\title{
ON THE NUMBER OF FACETS OF THREE-DIMENSIONAL DIRICHLET STEREOHEDRA IV: QUARTER CUBIC GROUPS
}

\author{
PILAR SABARIEGO AND FRANCISCO SANTOS
}

\begin{abstract}
In this paper we finish the intensive study of three-dimensional Dirichlet stereohedra started by the second author and D. Bochiş, who showed that they cannot have more than 80 facets, except perhaps for crystallographic space groups in the cubic system.

Taking advantage of the recent, simpler classification of three-dimensional crystallographic groups by Conway, Delgado-Friedrichs, Huson and Thurston, in a previous paper we proved that Dirichlet stereohedra for any of the 27 "full" cubic groups cannot have more than 25 facets. Here we study the remaining "quarter" cubic groups. With a computer-assisted method, our main result is that Dirichlet stereohedra for the 8 quarter groups, hence for all threedimensional crystallographic groups, cannot have more than 92 facets.
\end{abstract}

\section{INTRODUCTION}

This is the last in a series of four papers (see [1, 2, 17]) devoted to bounds on the number of facets that Dirichlet stereohedra in Euclidean 3-space can have.

A stereohedron is any bounded convex polyhedron which tiles the space by the action of some crystallographic group. A Dirichlet stereohedron for a certain crystallographic group $G$ is the Voronoi region $\operatorname{Vor}_{G p}(p)$ of a point $p \in \mathbb{R}^{3}$ in the Voronoi diagram of an orbit $G p$.

The study of the maximum number of facets for stereohedra is related to Hilbert's 18th problem, "Building up the space with congruent polyhedra" (see [12, 16]). Bieberbach (1910) and Reinhardt (1932) answered completly the first two of Hilbert's specific questions, but other problems related to monohedral tessellations (i. e., tessellations whose tiles are congruent) remain open. An exhaustive account of this topic appeared in a survey article by Grünbaum and Shephard [11, where our problem, to determine the maximum number of facets - or, at least, a "good" upper bound-for Dirichlet stereohedra in $\mathbb{R}^{3}$, is mentioned as an important one. Previous results on this problem are:

- The fundamental theorem of stereohedra (Delaunay, 1961 [6]) asserts that a stereohedron of dimension $d$ for a crystallographic group $G$ with a aspects cannot have more than $2^{d}(a+1)-2$ facets. The number of aspects of a crystallographic group $G$ is the index of its translational subgroup. Delone's bound for three-dimensional groups, which have up to 48 aspects, is 390 facets.

- The three-dimensional stereohedron with the maximum number of facets known so far was found in 1980 by P. Engel (see [7] and [11, p. 964]). It is a Dirichlet stereohedron with 38 facets, for the cubic group $I 4_{1} 32$, with 24 aspects.

Research partially supported by the Spanish Ministry of Education and Science, grant number MTM2008-04699-C03-02. 


\begin{tabular}{|c|c|c|c|c|c|c|c|}
\hline$|G: Q|$ & Aspects & Group & \multicolumn{5}{|c|}{ Our bounds } \\
\hline & & & $(1)$ & $(2)$ & $(3)$ & $(4)$ & Final \\
\hline 8 & 48 & $\mathcal{N}(Q)=I \frac{4_{1}}{g} \overline{3} \frac{2}{d}$ & 519 & 155 & 100 & 68 & $\mathbf{6 8}$ \\
\hline \multirow{3}{*}{4} & \multirow{3}{*}{24} & $I 4_{1} 32$ & 264 & 96 & 55 & & $\mathbf{5 5}$ \\
\cline { 3 - 8 } & & $I \overline{4} 3 d$ & 257 & 78 & & 76 & $\mathbf{7 6}$ \\
\cline { 3 - 8 } & 24 & $I \frac{2}{g} 3$ & 260 & 77 & & 57 & $\mathbf{5 7}$ \\
\hline \multirow{3}{*}{2} & 12 & $P 4_{1} 32$ & 135 & & 92 & & $\mathbf{9 2}$ \\
\cline { 3 - 8 } & 24 & $I 2^{\prime} 3$ & 131 & 48 & & 46 & $\mathbf{4 6}$ \\
\hline 1 & 12 & $P \frac{2_{1}}{a} \overline{3}$ & 132 & & & 86 & $\mathbf{8 6}$ \\
\hline & & $Q=P 2_{1} 3$ & 69 & & & & $\mathbf{6 9}$ \\
\hline
\end{tabular}

(1) Bounds after processing triad rotations.

(2) Bounds after diad rotations with axes parallel to the coordinate axes.

(3) Bounds after diagonal diad rotations.

(4) Bounds after intersecting with planar projections.

TABLE 1. Bounds for the number of facets of Dirichlet stereohedra of quarter cubic groups

There is agreement among the experts (see [7, page 214], [11, page 960], 18, page 50]) that Engel's sterohedron is much closer than Delone's upper bound to having the maximum possible number of facets. Our results confirm this.

In 2000, the second author and D. Bochiş gave upper bounds for the number of facets of Dirichlet stereohedra. They did this by dividing the 219 affine conjugacy classes of three-dimensional crystallographic groups into three blocks, and using different tools for each. Their main results are:

- Within the 100 crystallographic groups which contain reflection planes, the exact maximum number of facets is 18 [1].

- Within the 97 non-cubic crystallographic groups without reflection planes, they found Dirichlet stereohedra with 32 facets and proved that no one can have more than 80. Moreover, they got upper bounds of 50 and 38 for all but, respectively, 9 and 21 of the groups [2].

- They also considered cubic groups, but they were only able to prove an upper bound of 162 facets for them [3].

In 17 we improved the bound for 14 of the 22 cubic groups without reflections planes, the 14 "full groups":

Theorem 1.1. Dirichlet stereohedra for full cubic groups cannot have more than 25 facets.

In this paper we give an upper bound for the remaining cubic groups: the 8 "quarter groups". It has to be noted that to get these bounds, contrary to the ones in the previous papers of this series, computers are used. The upper bound we obtain for each quarter group is shown in Table 1. Columns (1) to (4) are the bounds obtained in different phases or our method, the column labeled "Final" is our final bound. Globally, we get the following.

Theorem 1.2. Dirichlet stereohedra for quarter cubic groups cannot have more than 92 facets.

For the sake of completeness, we include here the full list of other crystallographic groups for which the bounds proved in this series of papers is bigger than 38 (Table 2). This list is the same as Table 2 in [2]. 


\begin{tabular}{|c|c|c|}
\hline Group & Asp. & Bound \\
\hline$I \overline{4} c 2$ & 8 & $\mathbf{4 0}$ \\
$P \frac{4_{2}}{n} \frac{2}{g} \frac{2}{c}$ & 16 & $\mathbf{4 0}$ \\
$R \overline{3}$ & 6 & $\mathbf{4 2}$ \\
$R 32$ & 6 & $\mathbf{4 2}$ \\
$R 3 c$ & 6 & $\mathbf{4 2}$ \\
$I 4_{1} c d$ & 8 & $\mathbf{4 4}$ \\
$P 3_{1} 2$ & 6 & $\mathbf{4 8}$ \\
\hline
\end{tabular}

\begin{tabular}{|c|c|c|}
\hline Group & Asp. & Bound \\
\hline$P 3_{1} 12$ & 6 & $\mathbf{4 8}$ \\
$P 6_{1}$ & 6 & $\mathbf{4 8}$ \\
$P 4_{1} 22$ & 8 & $\mathbf{5 0}$ \\
$C \frac{2}{a} \frac{2}{c} \frac{2}{c}$ & 8 & $\mathbf{5 0}$ \\
$I \frac{2}{a} \frac{2}{c} \frac{2}{c}$ & 8 & $\mathbf{5 0}$ \\
$P 4_{1} 2_{1} 2$ & 8 & $\mathbf{6 4}$ \\
$I \frac{4_{1}}{g}$ & 8 & $\mathbf{7 0}$
\end{tabular} \mid \begin{tabular}{c|c|c|c|}
$I 4_{1} 22$ & 8 & $\mathbf{7 0}$ \\
$I \overline{4} 2 d$ & 8 & $\mathbf{7 0}$ \\
$F \frac{2}{d} \frac{2}{d} \frac{2}{d}$ & 8 & $\mathbf{7 0}$ \\
$P 6_{2} 22$ & 12 & $\mathbf{7 8}$ \\
$P 6_{1} 22$ & 12 & $\mathbf{7 8}$ \\
$R \overline{3} \frac{2}{c}$ & 12 & $\mathbf{7 9}$ \\
$I \frac{4_{1}}{g} \frac{2}{c} \frac{2}{d}$ & 16 & $\mathbf{8 0}$ \\
\hline
\end{tabular}

TABLE 2. Non-cubic groups where our upper bound is larger than 38

Theorem 1.3. Three dimensional Dirichlet stereohedra cannot have more than 92 facets. They can possibly have more than 38 facets only in one of the 29 groups listed in tables 1 and 2 .

\section{Preliminaries And outline}

2.1. "Full" and "quarter" cubic groups. Our division of cubic groups into "full" and "quarter" ones comes from the recent classification of three-dimensional crystallographic groups developed in 5 by Conway et al. They divide crystallographic groups into "reducible" and "irreducible", were irreducible groups are those that do not have any invariant direction. It turns out that they coincide with the cubic groups of the classical classification. Conway et al. define odd subgroup of an irreducible group $G$ as the one generated by the rotations of order three, and show that:

Theorem 2.1 (Conway et al. [5]). (1) There are only two possible odd subgroups of cubic groups, that we denote $F$ and $Q$.

(2) Both $F$ and $Q$ are normal in $\operatorname{Isom}\left(\mathbb{R}^{3}\right)$. Hence, every cubic group lies between its odd subgroup and the normalizer $\mathcal{N}(F)$ and $\mathcal{N}(Q)$ of it.

The second property reduces the enumeration of cubic space groups to the enumeration, up to conjugacy, of subgroups of the two finite groups $\mathcal{N}(F) / F$ and $\mathcal{N}(Q) / Q \cdot \mathcal{N}(Q) / Q$ is dihedral of order 8 and $\mathcal{N}(F) / F$ has order 16 and contains a dihedral subgroup of index 2 .

The main difference between $F$ and $Q$ is that $Q$ only contains triad rotations whose axes are mutually disjoint, while some triad rotation axes in $F$ intersect one another. $Q$ is a subgroup of $F$ of order four and because of that Conway et al. call full groups those with odd subgroup equal to $F$ and quarter groups those with odd subgroup equal to $Q$ : Quarter groups contain only a quarter of the possible rotation axes. There are 27 full groups (14 of them without reflection planes) and 8 quarter groups (none with reflection planes, because $\mathcal{N}(Q)$ does not contain reflections).

2.2. The structure of quarter cubic groups. Throughout the paper we use the International Crystallographic Notation for three-dimensional crystallographic groups; see, e. g., [15.

All the quarter cubic groups have the same odd group $Q$, a group of type $P 2_{1} 3$. By definition, $Q$ is generated by triad rotations. More precisely, through each point $(x, y, z) \in(\mathbb{Z} / 2)^{3}$ exactly one rotation axis passes, with vector: 


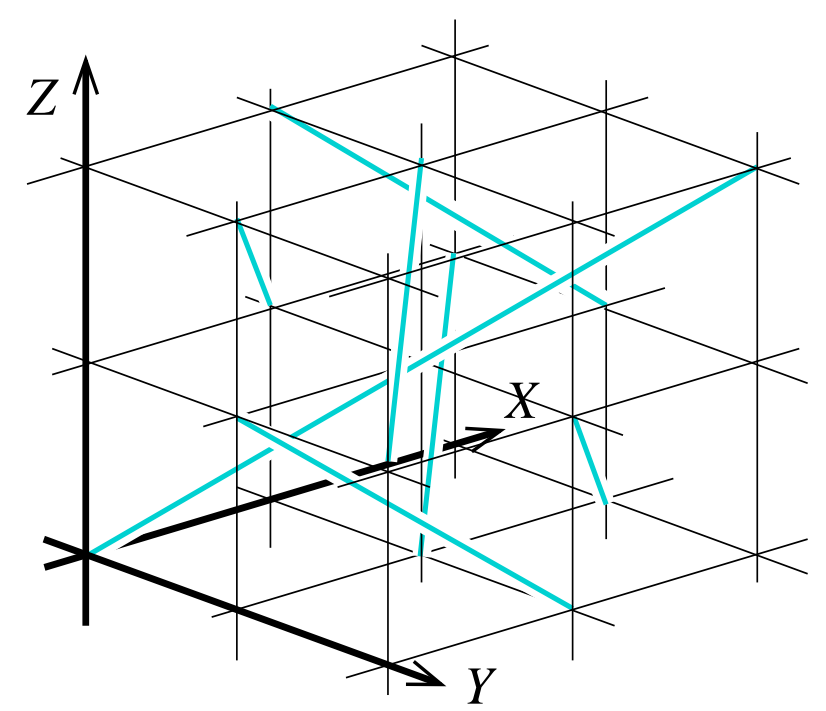

Figure 1. The body-centered cubic lattice $I$. The triad rotations (in grey) together with translations of length two in the coordinate directions generate $Q$

$$
\begin{aligned}
& (1,1,1) \text { if } x \equiv y \equiv z(\bmod 1) \\
& (-1,1,1) \text { if } y \not \equiv x \equiv z(\bmod 1) \\
& (1,-1,1) \text { if } z \not \equiv x \equiv y(\bmod 1) \\
& (1,1,-1) \text { if } x \neq \equiv y \equiv z(\bmod 1)
\end{aligned}
$$

In other words, exactly one of the four diagonals of each primitive cubic cell of the lattice $(\mathbb{Z} / 2)^{3}$ is a rotation axis (see Fig 1 ). The translational subgroup of $Q$ is a cubic primitive lattice with vectors of length one. $\mathcal{N}(Q)$ is the group of symmetries of the set of triad rotation axes. It is a group of type $I \frac{4_{1}}{g} \overline{3} \frac{2}{d}$, and its translational subgroup is a body centered lattice generated by the vectors $\left( \pm \frac{1}{2}, \pm \frac{1}{2}, \pm \frac{1}{2}\right)$.

$\mathcal{N}(Q) / Q$ has order 8 . In fact, it is isomorphic to the dihedral group $D_{8}$. Hence there are 8 groups between $\mathcal{N}(Q)$ and $Q$, including both. Their lattice is drawn in Figure 2, In Figure 2, we represent them graphically with the conventions of [15: for each group, the intersection of a generic orbit with the primitive cell $[0,1]^{3}$ is considered. Because all the groups contain the triad rotation on the diagonal axis $x=y=z$, only a third of the orbit is necessary in order to describe the group. E. g., the orbit with respect to the subgroup that sends horizontal planes to horizontal planes. This is what is drawn in the figures, projected over the $X Y$ plane.

The full dot near the origin represents a base point $(a, b, c)$ with $0 \leq a, b, c \leq 1 / 4$. Next to each of the other orbit points there is a number $h \in\{0,1 / 4,1 / 2,3 / 4\}$, omitted whenever it equals 0 . If an orbit point is represented as a "full dot", then its $z$-coordinate equals $c+h$. If it is represented as an "empty dot", then it equals $h-c$ (or $1-c$, if $h$ is zero). For extra understanding of the group, each dot is drawn dark or light depending on whether it is obtained from the base point by an orientation preserving or an orientation reversing isometry in $G$.

2.3. Outline of our method. Let $G$ be one of the eight quarter groups and let $p \in \mathbb{R}^{3}$ be a base point for an orbit $G p$, so that the Dirichlet stereohedron we want to study is the closed Voronoi region $\operatorname{Vor}_{G p}(p)$. There is no loss of generality in assuming that $p$ has trivial stabilizer, since Koch [13, 14, completely classified cubic orbits with non-trivial stabilizer (more generally, those with less than 3 degrees of 


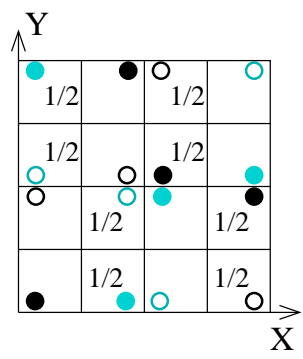

$I \frac{2}{g} \overline{3}$

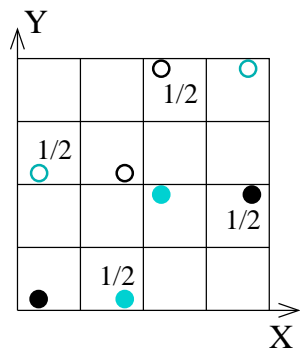

$P \frac{22_{1}}{a} \overline{3}$

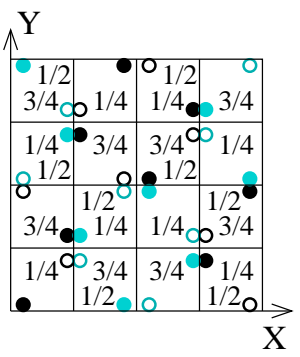

$\mathcal{N}(Q)=I \frac{4_{1}}{g} \overline{3} \frac{2}{d}$

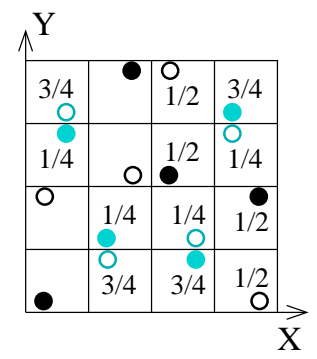

$I \overline{4} 3 d$

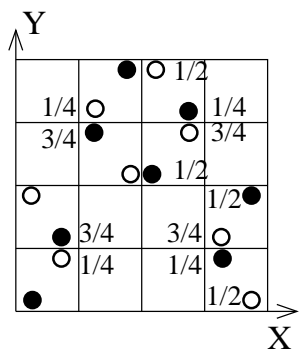

$I 4_{1} 32$

$P 4_{1} 32$

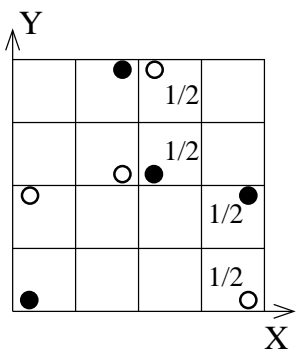

$I 2^{\prime} 3$

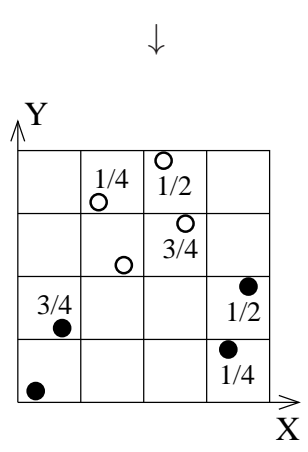

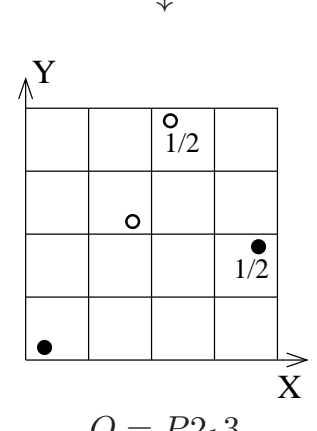

$$
Q=P 2_{1} 3
$$

FIgURE 2. The eight quarter groups.

freedom) and showed that they produce Dirichlet stereohedra with at most 23 facets. 
If $p$ has trivial stabilizer, then a small perturbation of its coordinates can only increase the number of facets [17, Lemma 3.1], so we assume $p$ to be sufficiently generic.

Our method is as follows: We consider a certain tessellation $\mathcal{T}$ of the threedimensional Euclidean space, which we call the auxiliary tessellation. For each tile $T$ of $\mathcal{T}$ we call extended Voronoi region of $T$ any region $\operatorname{VorExt}_{G} T$ with the following property:

$$
\forall q \in T, \quad \operatorname{Vor}_{G q}(q) \subseteq \operatorname{VorExt}_{G}(T) .
$$

The extended Voronoi region is not uniquely defined. Part of our work is to compute one that is as small as possible, for each tile $T$.

Now, if $T_{0}$ is the tile of $\mathcal{T}$ that contains the base point $p$, we call influence region of $T_{0}$ (or, of $p$ ) the union of all the tiles of $\mathcal{T}$ whose extended Voronoi regions meet $\operatorname{VorExt}_{G}\left(T_{0}\right)$ in their interiors. We denote it by $\operatorname{Infl}_{G}\left(T_{0}\right)$. Observe that, strictly speaking, $\operatorname{Infl}_{G}\left(T_{0}\right)$ depends not only on $G$, but also on $\mathcal{T}$ and on our particular choice of extended Voronoi regions. It is easy to prove:

Theorem 2.2. If $T_{0}$ is the tile containing $p$, then all the elements of $G p$ that are neighbors of $p$ in the Voronoi diagram $\operatorname{Vor}_{G p}(p)$ lie in $\operatorname{Infl}_{G}\left(T_{0}\right)$.

Proof. See 3 .

That is, to get a bound on the number of facets of Dirichlet stereohedra for base points in $T_{0}$ we only need to count, or bound, $\left|G p \cap \operatorname{Infl}_{G}\left(T_{0}\right)\right|$.

The above "sketch of a method" is impossible to implement directly. It involves the computation of an infinite number of extended Voronoi regions and influence regions. The key property that allows us to convert this into a finite computation is the following easy lemma:

Lemma 2.3. Let $\mathcal{N}(G)$ denote the normalizer of a crystallographic group $G$. If $T_{1}$ and $T_{2}=\rho T_{1}$ are tiles related by a transformation $\rho \in \mathcal{N}(G)$, then $\operatorname{VorExt}_{G}\left(T_{2}\right)=$ $\rho \operatorname{VorExt}_{G}\left(T_{1}\right)$ and $\operatorname{Infl}_{G}\left(T_{2}\right)=\rho \operatorname{Infl}_{G}\left(T_{1}\right)$.

Proof. It follows from the fact that $\operatorname{Vor}_{G \rho(q)}(\rho(q))=\rho\left(\operatorname{Vor}_{G q}(q)\right)$ for every point $q$, if $\rho$ is in the normalizer $\mathcal{N}(G)$ of $G$.

So, what we do is to use an auxiliary tessellation whose tiles lie in a small number of classes modulo the normalizer of $G$. Only this number of influence regions needs to be computed.

2.4. Computation of extended Voronoi regions. The basic idea for our computation of extended Voronoi regions is that each translation and rotation present in $G$ implies that a certain region of space can be excluded from $\operatorname{VorExt}_{G}\left(T_{0}\right)$.

Lemma 2.4. Let $T_{0}$ be a convex domain and let $\rho$ be a rotation in $G$ with axis $\ell$ and rotation angle $\alpha$. Assume that $\ell$ does not intersect $T_{0}$. Let $H_{1}$ and $H_{2}$ be the two support half-planes of $T_{0}$ with border in $\ell$. Let $H_{1}^{\prime}$ and $H_{2}^{\prime}$ be the half-planes obtained by rotating $H_{1}$ and $H_{2}$ "away from $T_{0}$ " with angles $\pm \alpha / 2$ and axis $\ell$.

Then, for every $p \in T_{0}$, the Dirichlet region $\operatorname{Vor}_{G p}(p)$ is contained in the (perhaps non-convex) dihedral region bounded by $H_{1}^{\prime} \cup H_{2}^{\prime}$ and containing $T_{0}$.

Proof. In fact, something stronger is $\operatorname{true} \operatorname{Vor}_{G p}(p)$ is contained in the dihedral sector with axis in $\ell$, angle $\alpha$, and centered at $p$. This follows from the fact that the two facets of this dihedron are parts of the bisectors of $p$ and $\rho p$ and of $p$ and $\rho^{-1} p$, respectively. See the left part of Figure 3 . 

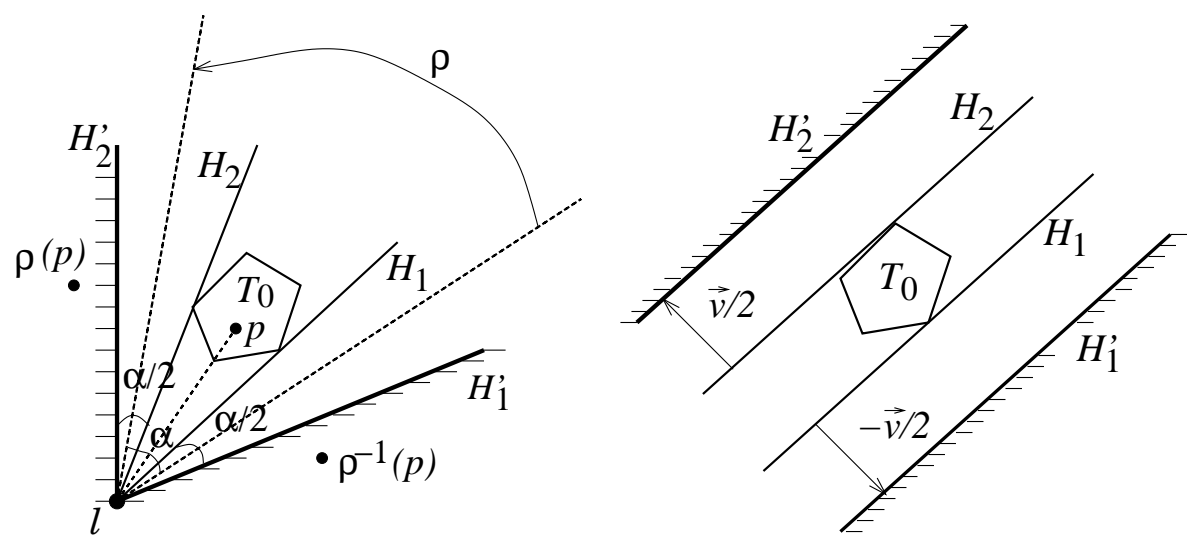

FiguRE 3. Situations in the Lemmas 2.4 and 2.5

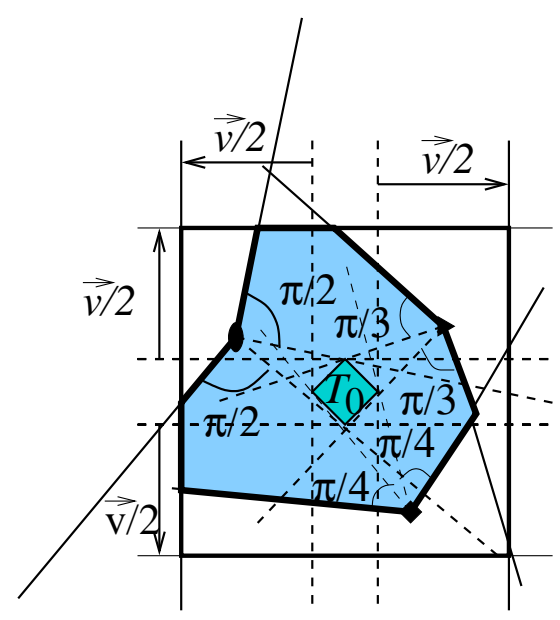

Figure 4. Extended Voronoi region induced by three rotations and two translations

Lemma 2.5. Let $T_{0}$ be a convex domain and let $\vec{v}$ be the vector of some translation in $G$. Let $H_{1}$ and $H_{2}$ be the two support planes of $T_{0}$ orthogonal to $\vec{v}$. Let $H_{1}^{\prime}$ and $H_{2}^{\prime}$ be the planes obtained translating $H_{1}$ and $H_{2}$ "away from $T_{0}$ " by the vectors $\pm \vec{v} / 2$.

Then, for every $p \in T_{0}$, the Dirichlet region $\operatorname{Vor}_{G p}(p)$ is contained in the strip between $H_{1}^{\prime}$ and $H_{2}^{\prime}$.

Proof. Similar to the previous one. See the right part of Figure 3

So, our method for constructing extended Voronoi regions consists in identifying a certain number of rotations and translations in $G$ and taking as $\operatorname{VorExt}_{G}\left(T_{0}\right)$ the intersection of the regions allowed in Lemmas 2.4 and 2.5. Figure 4 shows an example of what we mean, in which we consider three rotations and two translations in the plane.

The problem with this approach is that the regions obtained in Lemma 2.4 may not be convex. Observe that we are going to use more than 250 rotations (see Tables 9 10, 11 and 12). So, in principle, to compute an extended Voronoi region we would need to intersect 250 non-convex regions, which is an extremely hard computational problem (see, e. g., [8]). 


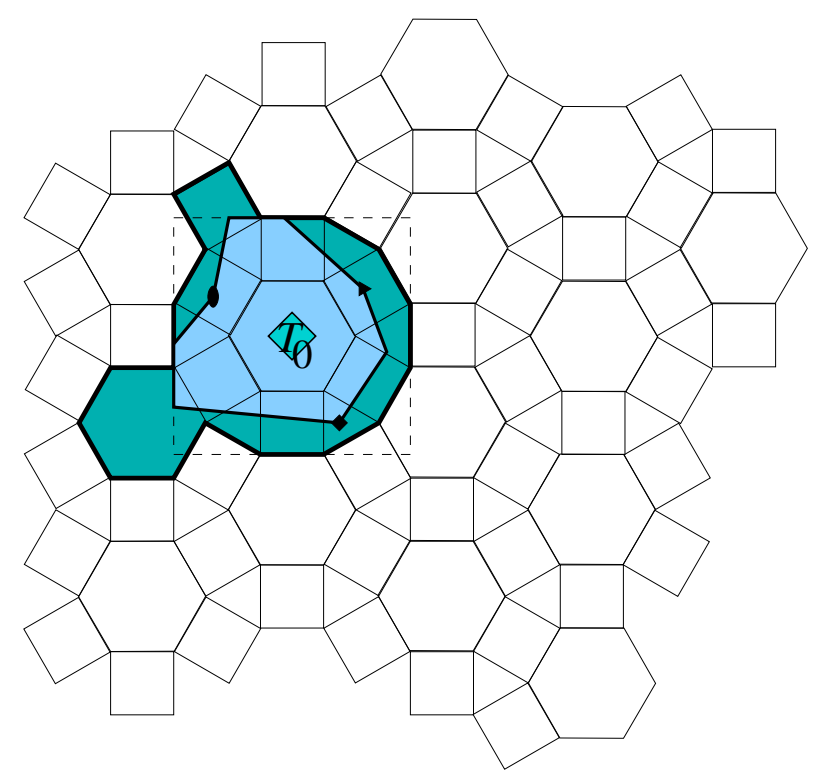

FiguRE 5. Extended Voronoi region over a tessellation

To avoid this we do the following, at the expense of getting a slightly larger (hence, for our purposes worse) extended Voronoi region: instead of obtaining the extended Voronoi region directly as an intersection, we obtain it as a union of tiles of the same auxiliary tessellation $\mathcal{T}$. That is to say:

(1) We first identify a finite (but large) population of tiles in $\mathcal{T}$ that form themselves an extended Voronoi region (that is, which are guaranteed to contain $\operatorname{Vor}_{G p}(p)$ for every $\left.p \in T_{0}\right)$.

(2) We then process one by one the rotations and translations we are interested in, and at each step discard from our initial population the tiles that do not intersect the corresponding region of Lemma 2.4 or Lemma 2.5.

What we eventually obtain with this method is the set of tiles of $\mathcal{T}$ that intersect the region of Figure 4. See Figure 5.

The reader may wonder why step (2) above is computationally not so hard, since it again involves the intersection of non-convex regions. The reason is that now we intersect only two such regions at a time (one tile and one strip or dihedron) and do not need to intersect the resulting set with anything else. Also (although less important), we do not really need to compute an intersection but only to check its emptyness.

\section{Detailed discussion of the method}

3.1. The auxiliary tessellation. The first natural choice of an auxiliary tessellation would be a tessellation by fundamental domains of the normalizer $\mathcal{N}(G)$, or of any subgroup of it. In this way all the tiles are equivalent: By Lemma 2.3, in such a tessellation we would need to compute only one extended Voronoi region.

Since we have $\mathcal{N}(Q) \leq \mathcal{N}(G)$ for every quarter group, as a first step let us compute a fundamental domain of $\mathcal{N}(Q)$ (later, we will subdivide it further). In order to get a fundamental domain with some symmetries, we start with the Voronoi region of one of its degenerate orbits, namely the orbit with base point at the origin $(0,0,0)$. This orbit is a body centered cubic lattice of side $1 / 2$. Its Voronoi cell is 


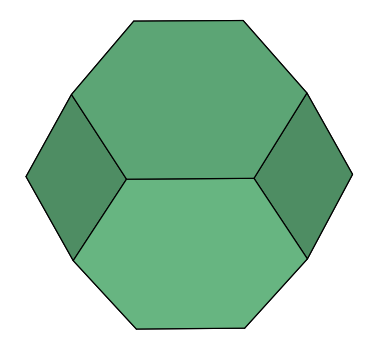

FiguRE 6. A truncated octahedron, the Voronoi cell of the body centered cubic lattice

a truncated octahedron (see Figure 6) whose 14 facets have supporting inequalities

$$
\pm x \leq \frac{1}{4}, \quad \pm y \leq \frac{1}{4}, \quad \pm z \leq \frac{1}{4}, \quad \pm x \pm y \pm z \leq \frac{3}{8} .
$$

The 24 vertices of the Voronoi cell are

$$
\begin{array}{lll}
\left( \pm \frac{1}{4}, \pm \frac{1}{8}, 0\right), & \left( \pm \frac{1}{4}, 0, \pm \frac{1}{8}\right), \quad\left(0, \pm \frac{1}{4}, \pm \frac{1}{8}\right), \\
\left( \pm \frac{1}{8}, \pm \frac{1}{4}, 0\right), & \left( \pm \frac{1}{8}, 0, \pm \frac{1}{4}\right), & \left(0, \pm \frac{1}{8}, \pm \frac{1}{4}\right) .
\end{array}
$$

The stabilizer of this truncated octahedron in $\mathcal{N}(Q)$ has order six. It is generated by the central symmetry (inversion) around the origin and the rotation of order three with axis in the line $x=z=y$. Hence, one way of obtaining a fundamental domain for $\mathcal{N}(Q)$ is to intersect the truncated octahedron with a sector of angle $\pi / 3$ with edge in that line. There are several inequivalent ways of doing it, but we choose the sector defined by the inequalities $x \leq y \leq z$.

In this way, we obtain as fundamental domain of $\mathcal{N}(Q)$ the polytope with the following 12 vertices:

$$
\begin{gathered}
\left(\frac{1}{8}, \frac{1}{8}, \frac{1}{8}\right) \\
\left(\frac{1}{4}, \frac{1}{16}, \frac{1}{16}\right) \quad\left(\frac{1}{4}, \frac{1}{8}, 0\right) \quad\left(\frac{3}{16}, \frac{3}{16}, 0\right) \\
\left(\frac{1}{4}, \frac{-1}{16}, \frac{-1}{16}\right) \quad\left(\frac{1}{4}, 0,-\frac{1}{8}\right) \quad\left(\frac{1}{8}, 0, \frac{-1}{4}\right) \quad\left(\frac{1}{16}, \frac{1}{16}, \frac{-1}{4}\right) \\
\left(0, \frac{-3}{16}, \frac{-3}{16}\right) \quad\left(0, \frac{-1}{8}, \frac{-1}{4}\right) \quad\left(\frac{-1}{16}, \frac{-1}{16}, \frac{-1}{4}\right) \\
\left(\frac{-1}{8}, \frac{-1}{8}, \frac{-1}{8}\right)
\end{gathered}
$$

This polytope is depicted on the left side of Figure 7 . It has eight facets: the two "big ones" in the planes $x=y$ and $y=z$ are pentagons and the other six are parts of facets of the truncated octahedron: two halves-of-squares, two halves-of-hexagons and two sixths-of-hexagons.

As we said above, to get better bounds, we further subdivide this fundamental domain of $\mathcal{N}(Q)$. We do it cutting with the coordinate planes $z=0, y=0$ and $x=0$. We get the following four sub-polytopes $A_{0}, B_{0}, C_{0}$ and $D_{0}$ (see Figure 7 ):

$$
A_{0}:=\operatorname{conv}\left((0,0,0),\left(\frac{1}{8}, \frac{1}{8}, \frac{1}{8}\right),\left(\frac{1}{4}, \frac{1}{16}, \frac{1}{16}\right),\left(\frac{1}{4}, \frac{1}{8}, 0\right),\left(\frac{3}{16}, \frac{3}{16}, 0\right),\left(\frac{1}{4}, 0,0\right)\right)
$$



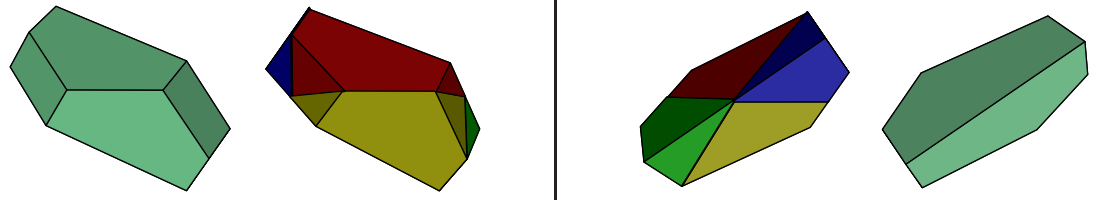

Figure 7. Two views of the fundamental domain of $\mathcal{N}(Q)$ and its subdivision into four prototiles, $A_{0}, B_{0}, C_{0}$ and $D_{0}$

$$
\begin{aligned}
B_{0}:= & \operatorname{conv}\left((0,0,0),\left(\frac{1}{4}, 0,0\right),\left(\frac{1}{4}, \frac{1}{8}, 0\right),\left(\frac{3}{16}, \frac{3}{16}, 0\right),\right. \\
& \left.\left(\frac{1}{4}, 0, \frac{-1}{8}\right),\left(\frac{1}{8}, 0, \frac{-1}{4}\right),\left(\frac{1}{16}, \frac{1}{16}, \frac{-1}{4}\right),\left(0,0, \frac{-1}{4}\right)\right) \\
C_{0}:= & \operatorname{conv}\left((0,0,0),\left(\frac{1}{4}, 0,0\right),\left(\frac{1}{4}, \frac{-1}{16}, \frac{-1}{16}\right),\left(\frac{1}{4}, 0, \frac{-1}{8}\right),\right. \\
& \left.\left(\frac{1}{8}, 0, \frac{-1}{4}\right),\left(0, \frac{-3}{16}, \frac{-3}{16}\right),\left(0, \frac{-1}{8}, \frac{-1}{4}\right),\left(0,0, \frac{-1}{4}\right)\right) \\
D_{0}:= & \operatorname{conv}\left((0,0,0),\left(0, \frac{-3}{16}, \frac{-3}{16}\right),\left(\frac{-1}{16}, \frac{-1}{16}, \frac{-1}{4}\right),\right. \\
& \left.\left(0, \frac{-1}{8}, \frac{-1}{4}\right),\left(\frac{-1}{8}, \frac{-1}{8}, \frac{-1}{8}\right),\left(0,0, \frac{-1}{4}\right)\right)
\end{aligned}
$$

Definition 3.1. From now on, the tessellation $\mathcal{T}$ of $\mathbb{R}^{3}$ obtained letting $\mathcal{N}(Q)$ act on the four "prototiles" $A_{0}, B_{0}, C_{0}$, and $D_{0}$ is called auxiliary tessellation.

3.2. The initial population of tiles. As said in Section 2.4 we compute extended Voronoi regions starting with a finite number of tiles of $\mathcal{T}$ whose union in guaranteed to contain $\operatorname{Vor}_{G p}(p)$ for every $p \in T_{0}$, and then eliminating as many of them as we can. As initial population of tiles, we take all the ones contained in the cube $[-1,1]^{3}$. That this is enough follows from the fact that the four prototiles are contained in the cube $[-1 / 4,1 / 4]^{3}$ and our group $Q$ contains the translations of length 1 in the three coordinate directions. Lemma 2.5 then implies that the extended Voronoi region (of any of the prototiles) is contained in the cube $[-3 / 4,3 / 4]^{3}$.

Since the four prototiles together form a fundamental domain of $\mathcal{N}(Q)$ and since the density of (any generic orbit of) $\mathcal{N}(Q)$ is 96 points per unit cube, in the initial population we have $96 \times 8=768$ tiles of each of the four types, and 3072 tiles in total.

In our implementation, each tile of $\mathcal{T}$ is characterized by its "type" ( $A, B, C$ or $D)$ and the transformation $\rho \in \mathcal{N}(Q)$ that sends the corresponding prototile $\left(A_{0}\right.$, 


\begin{tabular}{|c|c|c|c|c|c|c|c|c|c|c|c|c|c|c|c|c|}
\hline & \multicolumn{16}{|c|}{ Type $A$} \\
\hline \multirow{4}{*}{$\mathcal{C}_{1}$} & 1 & 0 & 0 & 0 & 1 & 0 & 0 & 0 & 1 & 0 & 0 & 0 & 1 & 0 & 0 & 0 \\
\hline & 0 & 1 & 0 & 0 & 1 & -1 & 0 & 0 & $1 / 2$ & -1 & 0 & 0 & $1 / 2$ & 1 & 0 & 0 \\
\hline & 0 & 0 & 1 & 0 & $1 / 2$ & 0 & -1 & 0 & $1 / 2$ & 0 & 1 & 0 & 1 & 0 & -1 & 0 \\
\hline & 0 & 0 & 0 & 1 & $1 / 2$ & 0 & 0 & 1 & 1 & 0 & 0 & -1 & $1 / 2$ & 0 & 0 & -1 \\
\hline \multirow{4}{*}{$\mathcal{C}_{2}$} & 1 & 0 & 0 & & 1 & 0 & 0 & 0 & 1 & 0 & 0 & 0 & 1 & 0 & 0 & 0 \\
\hline & $3 / 4$ & 0 & 1 & 0 & $1 / 4$ & 0 & -1 & 0 & $3 / 4$ & 0 & -1 & 0 & $1 / 4$ & 0 & 1 & 0 \\
\hline & $1 / 4$ & -1 & 0 & 0 & $1 / 4$ & 1 & 0 & 0 & $3 / 4$ & -1 & 0 & 0 & $3 / 4$ & 1 & 0 & 0 \\
\hline & $1 / 4$ & 0 & 0 & 1 & $3 / 4$ & 0 & 0 & 1 & $3 / 4$ & 0 & 0 & -1 & $1 / 4$ & 0 & 0 & -1 \\
\hline \multirow{4}{*}{$\mathcal{C}_{3}$} & 1 & 0 & 0 & 0 & 1 & 0 & 0 & 0 & 1 & 0 & 0 & 0 & 1 & $\overline{0}$ & 0 & 0 \\
\hline & 1 & -1 & 0 & 0 & 0 & 1 & 0 & 0 & $1 / 2$ & 1 & 0 & 0 & $1 / 2$ & - & 0 & 0 \\
\hline & 0 & 0 & 1 & 0 & $1 / 2$ & 0 & -1 & 0 & $1 / 2$ & 0 & 1 & 0 & 1 & 0 & -1 & 0 \\
\hline & $1 / 2$ & 0 & 0 & -1 & 1 & 0 & 0 & -1 & $1 / 2$ & 0 & 0 & 1 & 0 & 0 & 0 & 1 \\
\hline \multirow{4}{*}{$\mathcal{C}_{4}$} & 1 & 0 & 0 & 0 & 1 & 0 & 0 & 0 & 1 & 0 & 0 & 0 & 1 & $\overline{0}$ & 0 & 0 \\
\hline & $1 / 4$ & 0 & -1 & 0 & $3 / 4$ & 0 & 1 & 0 & $1 / 4$ & 0 & 1 & 0 & $3 / 4$ & 0 & -1 & 0 \\
\hline & $1 / 4$ & -1 & 0 & 0 & $1 / 4$ & 1 & 0 & 0 & $3 / 4$ & -1 & 0 & 0 & $3 / 4$ & 1 & 0 & 0 \\
\hline & $1 / 4$ & 0 & 0 & -1 & $3 / 4$ & 0 & 0 & -1 & $3 / 4$ & 0 & 0 & 1 & $1 / 4$ & 0 & 0 & 1 \\
\hline \multirow{4}{*}{$\mathcal{C}_{5}$} & 1 & 0 & 0 & 0 & 1 & 0 & 0 & 0 & 1 & 0 & 0 & 0 & 1 & $\overline{0}$ & 0 & 0 \\
\hline & $1 / 2$ & 1 & 0 & 0 & $1 / 2$ & -1 & 0 & 0 & 1 & -1 & 0 & 0 & 0 & 1 & 0 & 0 \\
\hline & 0 & 0 & 1 & 0 & $1 / 2$ & 0 & -1 & 0 & $1 / 2$ & 0 & 1 & 0 & 1 & 0 & -1 & 0 \\
\hline & 1 & 0 & 0 & -1 & $1 / 2$ & 0 & 0 & -1 & 0 & 0 & 0 & 1 & $1 / 2$ & 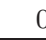 & 0 & 1 \\
\hline \multirow{4}{*}{$\mathcal{C}_{6}$} & 1 & 0 & 0 & 0 & 1 & 0 & 0 & 0 & 1 & 0 & 0 & 0 & 1 & & 0 & 0 \\
\hline & $1 / 4$ & 0 & 1 & 0 & $3 / 4$ & 0 & -1 & 0 & $1 / 4$ & 0 & -1 & 0 & $3 /$ & & 1 & 0 \\
\hline & $1 / 4$ & -1 & 0 & 0 & $1 / 4$ & 1 & 0 & 0 & $3 / 4$ & -1 & 0 & 0 & $3 /$ & & 0 & 0 \\
\hline & $3 / 4$ & 0 & 0 & -1 & $1 / 4$ & 0 & 0 & -1 & $1 / 4$ & 0 & 0 & 1 & $3 /$ & & 0 & 1 \\
\hline \multirow{4}{*}{$\mathcal{C}_{7}$} & 1 & 0 & 0 & 0 & 1 & 0 & 0 & 0 & 1 & 0 & 0 & 0 & 1 & 0 & 0 & 0 \\
\hline & $1 / 2$ & -1 & 0 & 0 & $1 / 2$ & 1 & 0 & 0 & 0 & 1 & 0 & 0 & 1 & -1 & 0 & 0 \\
\hline & 0 & 0 & 1 & 0 & $1 / 2$ & 0 & -1 & 0 & $1 / 2$ & 0 & 1 & 0 & 1 & 0 & -1 & 0 \\
\hline & $1 / 2$ & 0 & 0 & 1 & 0 & 0 & 0 & 1 & $1 / 2$ & 0 & 0 & -1 & 1 & 0 & 0 & -1 \\
\hline \multirow{4}{*}{$\mathcal{C}_{8}$} & 1 & 0 & 0 & 0 & 1 & 0 & 0 & 0 & 1 & 0 & 0 & 0 & 1 & 0 & 0 & 0 \\
\hline & $3 / 4$ & 0 & -1 & 0 & $1 / 4$ & 0 & 1 & 0 & $3 / 4$ & 0 & 1 & 0 & $1 / 4$ & 0 & -1 & 0 \\
\hline & $1 / 4$ & -1 & 0 & 0 & $1 / 4$ & 1 & 0 & 0 & $3 / 4$ & -1 & 0 & 0 & $3 / 4$ & 1 & 0 & 0 \\
\hline & $3 / 4$ & 0 & 0 & 1 & $1 / 4$ & 0 & 0 & 1 & $1 / 4$ & 0 & 0 & -1 & $3 / 4$ & 0 & 0 & -1 \\
\hline
\end{tabular}

TABLE 3. The 32 matrices of the isometries which send horizontal planes to horizontal planes for $A_{0}$

$B_{0}, C_{0}$ or $\left.D_{0}\right)$ to it. We compute the initial tiles of type, say, $A$, in the initial tessellation as follows:

- From the graphic representation of $\mathcal{N}(Q)$ in Figure 2 we read the 32 isometries in $\mathcal{N}(Q)$ that send horizontal planes to horizontal planes and send a base point $p \in[0,1 / 4]^{3}$ to lie in the unit cube $[0,1]^{3}$. The matrices of these isometries are shown in Table 3 . For future reference, these 32 matrices appear in eight subsets $\mathcal{C}_{i}, i=1, \ldots, 8$, representing the cosets modulo $Q$. Table 4 shows which cosets of isometries form each of the eight quarter groups.

- To these 32 isometries we apply the triad rotation with axis $x=y=z$, to obtain the 96 elements of $\mathcal{N}(Q)$ that send a point $p \in A_{0} \subset[0,1 / 4]^{3}$ to lie in $[0,1]^{3}$.

- Finally, we compose these 96 with the translations by the vectors $(0,0,0)$, $(-1,0,0),(0,-1,0),(0,0,-1),(-1,-1,0),(-1,0,-1),(0,-1,-1)$ and $(-1,-1,-1)$ in order to get all the tiles of type $A$ contained in $[-1,1]^{3}$.

With the tiles of the other types, $B, C$ and $D$, we work the same way, except for the fact that the prototiles are now not in $[0,1]^{3}$. For example: $B_{0}$ is in the unit cube $[0,1] \times[0,1] \times[-1,0]$. Still, to build our initial population of $B$-tiles, we want to start with the 32 horizontal transformations that send $B_{0}$ to lie in $[0,1]^{3}$. 


\begin{tabular}{|c|c|}
\hline Quarter groups & Cosets \\
\hline$I \frac{4_{1}}{g} \overline{3} \frac{2}{d}$ & $\mathcal{C}_{1}, \ldots, \mathcal{C}_{8}$ \\
\hline$I 4_{1} 32$ & $\mathcal{C}_{1}, \mathcal{C}_{2}, \mathcal{C}_{3}, \mathcal{C}_{4}$ \\
\hline$I \overline{4} 3 d$ & $\mathcal{C}_{1}, \mathcal{C}_{3}, \mathcal{C}_{6}, \mathcal{C}_{8}$ \\
\hline$I \frac{2}{g} \overline{3}$ & $\mathcal{C}_{1}, \mathcal{C}_{3}, \mathcal{C}_{5}, \mathcal{C}_{7}$ \\
\hline$P 4_{1} 32$ & $\mathcal{C}_{1}, \mathcal{C}_{2}$ \\
\hline$I 2^{\prime} 3$ & $\mathcal{C}_{1}, \mathcal{C}_{3}$ \\
\hline$P \frac{2_{1}}{a} \overline{3}$ & $\mathcal{C}_{1}, \mathcal{C}_{7}$ \\
\hline$P 2_{1} 3$ & $\mathcal{C}_{1}$ \\
\hline
\end{tabular}

TABLE 4. Cosets of isometries in each quarter group

\begin{tabular}{|c|c|c|c|c|c|c|c|c|c|c|c|c|c|c|c|c|c|c|c|}
\hline & \multicolumn{19}{|c|}{ Type $B$} \\
\hline \multirow{4}{*}{$\mathcal{C}_{1}$} & 1 & & & & 0 & & & & & & 1 & 0 & 0 & 0 & & & & & \\
\hline & ( & & & & 0 & & & & & & $1 / 2$ & -1 & 0 & 0 & & & & & \\
\hline & ( & & & & 0 & & & & & & $1 / 2$ & 0 & 1 & 0 & & & & & \\
\hline & & & & & 1 & & & & & & 0 & 0 & 0 & -1 & & & & & \\
\hline \multirow{4}{*}{$\mathcal{C}_{3}$} & & & & & & 1 & 0 & & & & & & & & 1 & 0 & 0 & & \\
\hline & & & & & & 0 & 1 & 0 & & 0 & & & & & $1 / 2$ & -1 & 10 & & 0 \\
\hline & & & & & & $1 / 2$ & 0 & - & & 0 & & & & & 1 & 0 & - & & 0 \\
\hline & & & & & & 0 & 0 & 0 & & -1 & & & & & 1 & 0 & & & 1 \\
\hline \multirow{4}{*}{$\mathcal{C}_{5}$} & 1 & & & 1 & & & & & & & 1 & 0 & 0 & & & & & & \\
\hline & $1 /$ & & & & 0 & & & & & & 1 & -1 & 0 & 0 & & & & & \\
\hline & 0 & & & & 0 & & & & & & $1 / 2$ & 0 & 1 & 0 & & & & & \\
\hline & 0 & & & & -1 & & & & & & 1 & 0 & 0 & 1 & & & & & \\
\hline \multirow{4}{*}{$\mathcal{C}_{7}$} & & & & & & 1 & c & & & 0 & & & & & 1 & 0 & & & \\
\hline & & & & & & $1 / 2$ & 1 & & & 0 & & & & & 1 & -1 & 0 & 0 & \\
\hline & & & & & & $1 / 2$ & ( & - & & 0 & & & & & 1 & 0 & -1 & 0 & \\
\hline & & & & & & 1 & ( & & & 1 & & & & & 0 & 0 & 0 & - & 1 \\
\hline
\end{tabular}

TABLE 5. The new matrices of the isometries which send horizontal planes to horizontal planes for $B_{0}$

These are the same 32 of Table 3 except some of them need to be composed with a suitable integer translation. The matrices that need modification are shown in Tables 5. 6, and 7. for types $B, C$ and $D$, respectively. As in case $A$, once we have got these isometries, we apply to them the triad rotations with axis $x=y=z$ and the translations by the vectors $(0,0,0),(-1,0,0),(0,-1,0),(0,0,-1),(-1,-1,0)$, $(-1,0,-1),(0,-1,-1)$ and $(-1,-1,-1)$ to get an initial population of $96 \times 8=768$ tiles of each type.

3.3. Cutting with translations and rotations. At this point we can say that we have already computed a single extended Voronoi region for the eight groups and the four prototiles: the cube $[-1,1]^{3}$ decomposed into $768 \times 4=3072$ tiles, 768 of each type $A, B, C$ or $D$. But this region is certainly not good enough for our purposes. Our next step is to apply Lemmas 2.4 and 2.5 to exclude from this initial population as many tiles as we can.

That is to say: Let $G$ be one of the eight quarter groups and let $T_{0}$ be one of the four prototiles $A_{0}, B_{0}, C_{0}$ and $D_{0}$. For each choice of $G$ and $T_{0}$ we do the following:

- Let $S$ be a set of rotations and translations present in $G$. Below we specify our choice of $S$ for each $G$, but essentially what we do is we take the translations of smaller length and the rotations of axis closer to the origin. 


\begin{tabular}{|c|c|c|c|c|c|c|c|c|c|c|c|c|c|c|c|c|}
\hline & \multicolumn{16}{|c|}{ Type $C$} \\
\hline \multirow{4}{*}{$\mathcal{C}_{1}$} & 1 & 0 & $0 \quad$ & 0 & & & & & 1 & 0 & 0 & 0 & 1 & 0 & 0 & 0 \\
\hline & 0 & 1 & $0 \quad$ & 0 & & & & & $1 / 2$ & -1 & 0 & 0 & $1 / 2$ & 1 & 0 & 0 \\
\hline & 1 & 0 & 1 & 0 & & & & & $1 / 2$ & 0 & 1 & 0 & 0 & 0 & -1 & 0 \\
\hline & 1 & 0 & 0 & 1 & & & & & 0 & 0 & 0 & -1 & $1 / 2$ & 0 & 0 & -1 \\
\hline \multirow{4}{*}{$\mathcal{C}_{3}$} & 1 & 0 & 0 & 0 & 1 & 0 & 0 & 0 & & & & & 1 & 0 & 0 & 0 \\
\hline & 1 & -1 & 0 & 0 & 0 & 1 & 0 & 0 & & & & & $1 / 2$ & -1 & 0 & 0 \\
\hline & 1 & 0 & 1 & 0 & $1 / 2$ & 0 & -1 & 0 & & & & & 0 & 0 & -1 & 0 \\
\hline & $1 / 2$ & 0 & 0 & -1 & 0 & 0 & 0 & -1 & & & & & 1 & 0 & 0 & 1 \\
\hline \multirow{4}{*}{$\mathcal{C}_{5}$} & 1 & 0 & 0 & 0 & & & & & 1 & 0 & 0 & 0 & 1 & 0 & 0 & 0 \\
\hline & $1 / 2$ & 1 & 0 & 0 & & & & & 1 & -1 & 0 & 0 & 0 & 1 & 0 & 0 \\
\hline & 1 & 0 & 1 & 0 & & & & & $1 / 2$ & 0 & 1 & 0 & 0 & 0 & -1 & 0 \\
\hline & 0 & 0 & 0 & -1 & & & & & 1 & 0 & 0 & 1 & $1 / 2$ & 0 & 0 & 1 \\
\hline \multirow{4}{*}{$\mathcal{C}_{7}$} & 1 & 0 & 0 & 0 & 1 & c & 0 & 0 & & & & & 1 & 0 & 0 & 0 \\
\hline & $1 / 2$ & -1 & 0 & 0 & $1 / 2$ & & 0 & 0 & & & & & 1 & -1 & 0 & 0 \\
\hline & 1 & 0 & 1 & 0 & $1 / 2$ & 0 & -1 & 0 & & & & & 0 & 0 & -1 & 0 \\
\hline & $1 / 2$ & 0 & 0 & 1 & 1 & 0 & 0 & 1 & & & & & 0 & 0 & 0 & -1 \\
\hline
\end{tabular}

TABLE 6 . The new matrices of the isometries which send horizontal planes to horizontal planes for $C_{0}$

\begin{tabular}{|c|cccc|cccc|ccccc|cccc|}
\hline & \multicolumn{11}{c|}{ Type $D$} \\
\hline \multirow{4}{*}{$\mathcal{C}_{1}$} & 1 & 0 & 0 & 0 & 1 & 0 & 0 & 0 & 1 & 0 & 0 & 0 & 1 & 0 & 0 & 0 \\
& 1 & 1 & 0 & 0 & 0 & -1 & 0 & 0 & $1 / 2$ & -1 & 0 & 0 & $1 / 2$ & 1 & 0 & 0 \\
& 1 & 0 & 1 & 0 & $1 / 2$ & 0 & -1 & 0 & $1 / 2$ & 0 & 1 & 0 & 0 & 0 & -1 & 0 \\
& 1 & 0 & 0 & 1 & $1 / 2$ & 0 & 0 & 1 & 0 & 0 & 0 & -1 & $1 / 2$ & 0 & 0 & -1 \\
\hline \multirow{4}{*}{$\mathcal{C}_{3}$} & 1 & 0 & 0 & 0 & 1 & 0 & 0 & 0 & & & & & 1 & 0 & 0 & 0 \\
& 0 & -1 & 0 & 0 & 1 & 1 & 0 & 0 & & & & & $1 / 2$ & -1 & 0 & 0 \\
& 1 & 0 & 1 & 0 & $1 / 2$ & 0 & -1 & 0 & & & & & 0 & 0 & -1 & 0 \\
& $1 / 2$ & 0 & 0 & -1 & 0 & 0 & 0 & -1 & & & & & 1 & 0 & 0 & 1 \\
\hline \multirow{4}{*}{$\mathcal{C}_{5}$} & 1 & 0 & 0 & 0 & & & & & 1 & 0 & 0 & 0 & 1 & 0 & 0 & 0 \\
& $1 / 2$ & 1 & 0 & 0 & & & & & 0 & -1 & 0 & 0 & 1 & 1 & 0 & 0 \\
& 1 & 0 & 1 & 0 & & & & & $1 / 2$ & 0 & 1 & 0 & 0 & 0 & -1 & 0 \\
& 0 & 0 & 0 & -1 & & & & & 1 & 0 & 0 & 1 & $1 / 2$ & 0 & 0 & 1 \\
\hline \multirow{5}{*}{$\mathcal{C}_{7}$} & 1 & 0 & 0 & 0 & 1 & 0 & 0 & 0 & 1 & 0 & 0 & 0 & 1 & 0 & 0 & 0 \\
& $1 / 2$ & -1 & 0 & 0 & $1 / 2$ & 1 & 0 & 0 & 1 & 1 & 0 & 0 & 0 & -1 & 0 & 0 \\
& 1 & 0 & 1 & 0 & $1 / 2$ & 0 & -1 & 0 & $1 / 2$ & 0 & 1 & 0 & 0 & 0 & -1 & 0 \\
& $1 / 2$ & 0 & 0 & 1 & 1 & 0 & 0 & 1 & $1 / 2$ & 0 & 0 & -1 & 0 & 0 & 0 & -1 \\
\hline
\end{tabular}

TABLE 7. The new matrices of the isometries which send horizontal planes to horizontal planes for $D_{0}$

- For each of the $768 \times 4$ tiles in the initial population and for each element $\rho \in S$, apply Lemma 2.4 (if $\rho$ is a rotation) or 2.5 (if $\rho$ is a translation) and, if the tile is fully contained in the region of space forbidden by the Lemma, then remove this tile from the extended Voronoi region.

There is a subtle point in this latter test. Let $T$ be a tile to which we can apply the test of Lemma, say, 2.4. The "forbidden region" is the unbounded dihedron defined by two half-spaces $H_{1}^{\prime}$ and $H_{2}^{\prime}$ (see Figure 3). What we test is whether each of the vertices of $T$ belongs to each of the two halfspaces. But the decision of whether the tile is discarded or not depends on whether the dihedron is convex or not. That is, whether the angle $\alpha$ of the 


\begin{tabular}{|c|c|}
\hline Sets & Isommetries \\
\hline$S_{1}$ & $\begin{array}{c}\text { Coordinate translations: } \\
( \pm 1,0,0),(0, \pm 1,0),(0,0, \pm 1)\end{array}$ \\
\hline$S_{2}$ & $\begin{array}{c}\text { Diagonal translations: } \\
( \pm 1 / 2, \pm 1 / 2, \pm 1 / 2)\end{array}$ \\
\hline$S_{3}$ & Triad rotations (Table 9$)$ \\
\hline$S_{4}$ & Diad rotations (Table [10) \\
\hline$S_{5}$ & Diad rotations (Table [1]) \\
\hline$S_{6}$ & Diad rotations (Table [12) \\
\hline
\end{tabular}

\begin{tabular}{|c|l|l|}
\hline Group & Transl. & Rotations \\
\hline$P 2_{1} 3$ & $S_{1}$ & $S_{3}$ \\
$P \frac{21}{a} \overline{3}$ & & \\
\hline$P 4_{1} 32$ & $S_{1}$ & $S_{3}, S_{6}$ \\
\hline$I \overline{4} 3 d$ & & \\
$I \frac{2}{g} \overline{3}$ & $S_{1}, S_{2}$ & $S_{3}, S_{4}$ \\
$I 2^{\prime} 3$ & & \\
\hline$I \frac{4_{1}}{g} \overline{3} \frac{2}{d}$ & $S_{1}, S_{2}$ & $S_{3}, S_{4}, S_{5}$ \\
$I 4_{1} 32$ & \\
\hline
\end{tabular}

TABLE 8. Transformations that we use in each quarter group

rotation $\rho$ plus the angle with which $T_{0}$ is seen from $l$ is bigger or smaller than 180 degrees:

- If this angle is bigger than 180 degrees (that is, if the forbidden region is convex) we discard the tile $T$ whenever "both half-spaces $H_{1}^{\prime}$ and $H_{2}^{\prime}$ contain all the vertices of $T$ ".

- If this angle is smaller than 180 degrees (that is, if the forbidden region is not convex) we discard the tile $T$ whenever "one of the half-spaces $H_{1}^{\prime}$ and $H_{2}^{\prime}$ contains all the vertices of $T$ ".

If $\rho$ is a translation, the second rule is always applied.

The particular translations and rotations that we use are the following ones. Tables 8, 9, 10, 11, and 12 list them more explicitly.

- The integer translations of length one appear in all the quarter groups. In the five groups with a body centered translational subgroup we also use the half-integer diagonal translations $( \pm 1 / 2, \pm 1 / 2, \pm 1 / 2)$.

- The triad rotations also appear in all the quarter groups. The set $S_{3}$ of triad rotations that we use are listed in Table 9. We have included the ones with axis closer to the prototile, since the ones that are not close will cut out only portions of the extended Voronoi region that were already cut by other, closer rotations. The decision on where to put the threshold is somehow heuristic but should not affect the result much. Actually, many of the rotations listed in our table turned out to be already superfluous.

- Table10 lists the set $S_{4}$ of diad rotations with axes parallel to the coordinate axes that we use in the groups $I \frac{4_{1}}{g} \overline{3} \frac{2}{d}, I 4_{1} 32, I \overline{4} 3 d, I \frac{2}{g} \overline{3}$ and $I 2^{\prime} 3$.

- Table11 lists the set $S_{5}$ of diad rotations with axes parallel to the diagonals of the faces of the unit cube that we use in the groups $I \frac{4_{1}}{g} \overline{3} \frac{2}{d}$ and $I 4_{1} 32$. In the group $P 4_{1} 32$ only some of these diad rotations are present. The list $S_{6}$ of them is in Table 12 .

In these four tables (Tables 9, 10, 11 and 12), the two entries in each column are a point and the direction of the rotation axis.

3.4. Planar projection. In the extended Voronoi region computed so far only rotations and translations present in our group are taken into account. If we wish to deal with other transformations (glide reflections, screw rotations, etc) we could state the analogues of Lemmas 2.4 and 2.5 for them, but the result would be too complicated, perhaps impractical.

Instead, let us consider the "horizontal subgroup" of our group $G$. That is, the subgroup $G_{z}$ that fixes the third coordinate. Each of the eight parts of Figure 8 shows the planar subgroup of the quarter group that occupies the same position in Figure 2. Only four different planar subgroups arise, of types $p 1, p 2, p g$ and $p g g$. 
Triad rotations

\begin{tabular}{cc|cc|cc}
\hline Point & Vector & Point & Vector & Point & Vector \\
\hline$(0,0,0)$ & $(1,1,1)$ & $(-1 / 2,0,0)$ & $(-1,-1,1)$ & $(0,1 / 2,0)$ & $(-1,1,1)$ \\
$(-1,0,0)$ & $(1,1,1)$ & $(1 / 2,0,0)$ & $(-1,-1,1)$ & $(0,-1 / 2,0)$ & $(-1,1,1)$ \\
$(1,0,0)$ & $(1,1,1)$ & $(1 / 2,1,0)$ & $(-1,-1,1)$ & $(-1,1 / 2,0)$ & $(-1,1,1)$ \\
$(0,-1,0)$ & $(1,1,1)$ & $(-1 / 2,1,0)$ & $(-1,-1,1)$ & $(-1,-1 / 2,0)$ & $(-1,1,1)$ \\
$(0,1,0)$ & $(1,1,1)$ & $(1 / 2,-1,0)$ & $(-1,-1,1)$ & $(1,1 / 2,0)$ & $(-1,1,1)$ \\
$(0,0,-1)$ & $(1,1,1)$ & $(-1 / 2,-1,0)$ & $(-1,-1,1)$ & $(1,-1 / 2,0)$ & $(-1,1,1)$ \\
$(0,0,1)$ & $(1,1,1)$ & $(-3 / 2,-1,0)$ & $(-1,-1,1)$ & $(-1,3 / 2,0)$ & $(-1,1,1)$ \\
$(0,0,1 / 2)$ & $(1,-1,1)$ & $(1 / 2,0,1)$ & $(-1,-1,1)$ & $(0,1 / 2,-1)$ & $(-1,1,1)$ \\
$(1,0,1 / 2)$ & $(1,-1,1)$ & $(-1 / 2,0,1)$ & $(-1,-1,1)$ & $(0,-1 / 2,-1)$ & $(-1,1,1)$ \\
$(0,0,-1 / 2)$ & $(1,-1,1)$ & $(1 / 2,0,-1)$ & $(-1,-1,1)$ & $(0,1 / 2,1)$ & $(-1,1,1)$ \\
$(1,0,-1 / 2)$ & $(1,-1,1)$ & $(-1 / 2,0,-1)$ & $(-1,-1,1)$ & $(0,-1 / 2,1)$ & $(-1,1,1)$ \\
$(0,1,-1 / 2)$ & $(1,-1,1)$ & $(0,0,3 / 2)$ & $(1,-1,1)$ & $(1,-1 / 2,0)$ & $(-1,1,1)$ \\
$(0,1,1 / 2)$ & $(1,-1,1)$ & $(0,0,-3 / 2)$ & $(1,-1,1)$ & $(1,1 / 2,0)$ & $(-1,1,1)$ \\
& & $(1 / 2,3 / 2,0)$ & $(1,-1,1)$ & $(-1,1 / 2,0)$ & $(-1,1,1)$
\end{tabular}

TABLE 9. A set $S_{3}$ of triad rotations in $Q$ and, therefore, in all the quarter groups

Diad rotations parallel to the coordinate axes

\begin{tabular}{cc|cc|cc}
\hline Point & Vector & Point & Vector & Point & Vector \\
\hline$(0,-3 / 4,-1 / 2)$ & $(1,0,0)$ & $(-1 / 2,0,-3 / 4)$ & $(0,1,0)$ & $(-3 / 4,-1 / 2,0)$ & $(0,0,1)$ \\
$(0,-3 / 4,0)$ & $(1,0,0)$ & $(-1 / 2,0,-1 / 4)$ & $(0,1,0)$ & $(-3 / 4,0,0)$ & $(0,0,1)$ \\
$(0,-3 / 4,1 / 2)$ & $(1,0,0)$ & $(-1 / 2,0,1 / 4)$ & $(0,1,0)$ & $(-3 / 4,1 / 2,0)$ & $(0,0,1)$ \\
$(0,-1 / 4,-1 / 2)$ & $(1,0,0)$ & $(-1 / 2,0,3 / 4)$ & $(0,1,0)$ & $(-1 / 4,-1 / 2,0)$ & $(0,0,1)$ \\
$(0,-1 / 4,0)$ & $(1,0,0)$ & $(0,0,-3 / 4)$ & $(0,1,0)$ & $(-1 / 4,0,0)$ & $(0,0,1)$ \\
$(0,-1 / 4,1 / 2)$ & $(1,0,0)$ & $(0,0,-1 / 4)$ & $(0,1,0)$ & $(-1 / 4,1 / 2,0)$ & $(0,0,1)$ \\
$(0,1 / 4,-1 / 2)$ & $(1,0,0)$ & $(0,0,1 / 4)$ & $(0,1,0)$ & $(1 / 4,-1 / 2,0)$ & $(0,0,1)$ \\
$(0,1 / 4,0)$ & $(1,0,0)$ & $(0,0,3 / 4)$ & $(0,1,0)$ & $(1 / 4,0,0)$ & $(0,0,1)$ \\
$(0,1 / 4,1 / 2)$ & $(1,0,0)$ & $(1 / 2,0,-3 / 4)$ & $(0,1,0)$ & $(1 / 4,1 / 2,0)$ & $(0,0,1)$ \\
$(0,3 / 4,-1 / 2)$ & $(1,0,0)$ & $(1 / 2,0,-1 / 4)$ & $(0,1,0)$ & $(3 / 4,-1 / 2,0)$ & $(0,0,1)$ \\
$(0,3 / 4,0)$ & $(1,0,0)$ & $(1 / 2,0,1 / 4)$ & $(0,1,0)$ & $(3 / 4,0,0)$ & $(0,0,1)$ \\
$(0,3 / 4,1 / 2)$ & $(1,0,0)$ & $(1 / 2,0,3 / 4)$ & $(0,1,0)$ & $(3 / 4,1 / 2,0)$ & $(0,0,1)$
\end{tabular}

TABLE 10. A set $S_{4}$ of diad rotations parallel to the coordinate axes that appear in the groups $I \frac{4_{1}}{g} \overline{3} \frac{2}{d}, I 4_{1} 32, I 43 d, I \frac{2}{g} \overline{3}$ and $I 2^{\prime} 3$

As it turns out, extended Voronoi regions (and influence regions) for these four groups were computed in [3] and [1]. Our idea is to take advantage of the following simple fact:

Lemma 3.2. Let $\pi: \mathbb{R}^{3} \rightarrow \mathbb{R}^{2}$ denote the vertical projection, omitting the third coordinate. Let $T_{0}$ be a prototile and let $G$ be a crystallographic group. Let $G_{z}$ denote the horizontal subgroup of $G$, that is the set of transformations that send each horizontal plane to itself. Then:

$$
\pi\left(\operatorname{VorExt}_{G}\left(T_{0}\right)\right) \subseteq \operatorname{VorExt}_{G_{z}}\left(\pi\left(T_{0}\right)\right)
$$

Proof. The only thing to prove is the same formula for Voronoi regions. That is, for every base point $p \in T_{0}$ we need to show that:

$$
\pi\left(\operatorname{Vor}_{G p}(p)\right) \subseteq \operatorname{Vor}_{G_{z}(\pi(p))}(\pi(p)) .
$$




\begin{tabular}{|c|c|c|c|c|c|}
\hline Point & Vector & Point & Vector & Point & Vector \\
\hline$(-7 / 4,0,1 / 8)$ & $(-1,1,0)$ & $(-5 / 4,0,3 / 8)$ & $(1,1,0)$ & $(1 / 8,-7 / 4,0)$ & $(0,-1,1)$ \\
\hline$(-7 / 4,0,5 / 8)$ & $(-1,1,0)$ & $(-5 / 4,0,7 / 8)$ & $(1,1,0)$ & $(5 / 8,-7 / 4,0)$ & $(0,-1,1)$ \\
\hline$(-5 / 4,0,3 / 8)$ & $(-1,1,0)$ & $(-3 / 4,0,1 / 8)$ & $(1,1,0)$ & $(3 / 8,-5 / 4,0)$ & $(0,-1,1)$ \\
\hline$(-5 / 4,0,7 / 8)$ & $(-1,1,0)$ & $(-3 / 4,0,5 / 8)$ & $(1,1,0)$ & $(7 / 8,-5 / 4,0)$ & $(0,-1,1)$ \\
\hline$(-3 / 4,0,1 / 8)$ & $(-1,1,0)$ & $(-1 / 4,0,3 / 8)$ & $(1,1,0)$ & $(1 / 8,-3 / 4,0)$ & $(0,-1,1)$ \\
\hline$(-3 / 4,0,5 / 8)$ & $(-1,1,0)$ & $(-1 / 4,0,7 / 8)$ & $(1,1,0)$ & $(5 / 8,-3 / 4,0)$ & $(0,-1,1)$ \\
\hline$(-1 / 4,0,3 / 8)$ & $(-1,1,0)$ & $(1 / 4,0,1 / 8)$ & $(1,1,0)$ & $(3 / 8,-1 / 4,0)$ & $(0,-1,1)$ \\
\hline$(-1 / 4,0,7 / 8)$ & $(-1,1,0)$ & $(1 / 4,0,5 / 8)$ & $(1,1,0)$ & $(7 / 8,-1 / 4,0)$ & $(0,-1,1)$ \\
\hline$(1 / 4,0,1 / 8)$ & $(-1,1,0)$ & $(3 / 4,0,3 / 8)$ & $(1,1,0)$ & $(1 / 8,1 / 4,0)$ & $(0,-1,1)$ \\
\hline$(1 / 4,0,5 / 8)$ & $(-1,1,0)$ & $(3 / 4,0,7 / 8)$ & $(1,1,0)$ & $(5 / 8,1 / 4,0)$ & $(0,-1,1)$ \\
\hline$(3 / 4,0,3 / 8)$ & $(-1,1,0)$ & $(5 / 4,0,1 / 8)$ & $(1,1,0)$ & $(3 / 8,3 / 4,0)$ & $(0,-1,1)$ \\
\hline$(3 / 4,0,7 / 8)$ & $(-1,1,0)$ & $(5 / 4,0,5 / 8)$ & $(1,1,0)$ & $(7 / 8,3 / 4,0)$ & $(0,-1,1)$ \\
\hline$(5 / 4,0,1 / 8)$ & $1,0)$ & $(7 / 4,0,3 / 8)$ & $(1,1,0)$ & $(1 / 8,5 / 4,0)$ & $(0,-1,1)$ \\
\hline$(5 / 4,0,5 / 8)$ & $(-1,1,0)$ & $(7 / 4,0,7 / 8)$ & $(1,1,0)$ & $(5 / 8,5 / 4,0)$ & $(0,-1,1)$ \\
\hline$(7 / 4,0,3 / 8)$ & $(-1,1,0)$ & $(-5 / 4,0,-1 / 8)$ & $(1,1,0)$ & $(3 / 8,7 / 4,0)$ & $(0,-1,1)$ \\
\hline$(7 / 4,0,7 / 8)$ & $(-1,1,0)$ & $(-5 / 4,0,-5 / 8)$ & $(1,1,0)$ & $(7 / 8,7 / 4,0)$ & $(0,-1,1)$ \\
\hline$(-7 / 4,0,-3 / 8)$ & $(-1,1,0)$ & $(-3 / 4,0,-3 / 8)$ & $(1,1,0)$ & $(-3 / 8,-7 / 4,0)$ & $(0,-1,1)$ \\
\hline$(-7 / 4,0,-7 / 8)$ & $(-1,1,0)$ & $(-3 / 4,0,-7 / 8)$ & $(1,1,0)$ & $(-7 / 8,-7 / 4,0)$ & $(0,-1,1)$ \\
\hline$(-5 / 4,0,-1 / 8)$ & $(-1,1,0)$ & $(-1 / 4,0,-1 / 8)$ & $(1,1,0)$ & $(-1 / 8,-5 / 4,0)$ & $(0,-1,1)$ \\
\hline$(-5 / 4,0,-5 / 8)$ & $(-1,1,0)$ & $(-1 / 4,0,-5 / 8)$ & $(1,1,0)$ & $(-5 / 8,-5 / 4,0)$ & $(0,-1,1)$ \\
\hline$(-3 / 4,0,-3 / 8)$ & $(-1,1,0)$ & $(1 / 4,0,-3 / 8)$ & $(1,1,0)$ & $(-3 / 8,-3 / 4,0)$ & $(0,-1,1)$ \\
\hline$(-3 / 4,0,-7 / 8)$ & $(-1,1,0)$ & $(1 / 4,0,-7 / 8)$ & $(1,1,0)$ & $(-7 / 8,-3 / 4,0)$ & $(0,-1,1)$ \\
\hline$(-1 / 4,0,-1 / 8)$ & $(-1,1,0)$ & $(3 / 4,0,-1 / 8)$ & $(1,1,0)$ & $(-1 / 8,-1 / 4,0)$ & $(0,-1,1)$ \\
\hline$(-1 / 4,0,-5 / 8)$ & $(-1,1,0)$ & $(3 / 4,0,-5 / 8)$ & $(1,1,0)$ & $(-5 / 8,-1 / 4,0)$ & $(0,-1,1)$ \\
\hline$(1 / 4,0,-3 / 8)$ & $(-1,1,0)$ & $(5 / 4,0,-3 / 8)$ & $(1,1,0)$ & $(-3 / 8,1 / 4,0)$ & $(0,-1,1)$ \\
\hline$(1 / 4,0,-7 / 8)$ & $(-1,1,0)$ & $(5 / 4,0,-7 / 8)$ & $(1,1,0)$ & $(-7 / 8,1 / 4,0)$ & $(0,-1,1)$ \\
\hline$(3 / 4,0,-1 / 8)$ & $(-1,1,0)$ & $(7 / 4,0,-1 / 8)$ & $(1,1,0)$ & $(-1 / 8,3 / 4,0)$ & $(0,-1,1)$ \\
\hline$(3 / 4,0,-5 / 8)$ & $(-1,1,0)$ & $(7 / 4,0,-5 / 8)$ & $(1,1,0)$ & $(-5 / 8,3 / 4,0)$ & $(0,-1,1)$ \\
\hline$(5 / 4,0,-3 / 8)$ & $(-1,1,0)$ & $(0,1 / 8,-7 / 4)$ & $(1,0,-1)$ & $(-3 / 8,5 / 4,0)$ & $(0,-1,1)$ \\
\hline$(5 / 4,0,-7 / 8)$ & $(-1,1,0)$ & $(0,1 / 8,-7 / 4)$ & $(1,0,-1)$ & $(-7 / 8,5 / 4,0)$ & $(0,-1,1)$ \\
\hline$(7 / 4,0,-1 / 8)$ & $(-1,1,0)$ & $(0,3 / 8,-5 / 4)$ & $(1,0,-1)$ & $(-1 / 8,7 / 4,0)$ & $(0,-1,1)$ \\
\hline$(7 / 4,0,-5 / 8)$ & $(-1,1,0)$ & $(0,7 / 8,-5 / 4)$ & $(1,0,-1)$ & $(-5 / 8,7 / 4,0)$ & $(0,-1,1)$ \\
\hline$(3 / 8,-5 / 4,0)$ & $(0,1,1)$ & $(0,1 / 8,-3 / 4)$ & $(1,0,-1)$ & $(0,3 / 8,-5 / 4)$ & $(1,0,1)$ \\
\hline$(7 / 8,-5 / 4,0)$ & $(0,1,1)$ & $(0,5 / 8,-3 / 4)$ & $(1,0,-1)$ & $(0,7 / 8,-5 / 4)$ & $(1,0,1)$ \\
\hline$(1 / 8,-3 / 4,0)$ & $(0,1,1)$ & $(0,3 / 8,-1 / 4)$ & $(1,0,-1)$ & $(0,1 / 8,-3 / 4)$ & $(1,0,1)$ \\
\hline$(5 / 8,-3 / 4,0)$ & $(0,1,1)$ & $(0,7 / 8,-1 / 4)$ & $(1,0,-1)$ & $(0,5 / 8,-3 / 4)$ & $(1,0,1)$ \\
\hline$(3 / 8,-1 / 4,0)$ & $(0,1,1)$ & $(0,1 / 8,1 / 4)$ & $(1,0,-1)$ & $(0,3 / 8,-1 / 4)$ & $(1,0,1)$ \\
\hline$(7 / 8,-1 / 4,0)$ & $(0,1,1)$ & $(0,5 / 8,1 / 4)$ & $(1,0,-1)$ & $(0,7 / 8,-1 / 4)$ & $(1,0,1)$ \\
\hline$(1 / 8,1 / 4,0)$ & $(0,1,1)$ & $(0,3 / 8,3 / 4)$ & $(1,0,-1)$ & $(0,1 / 8,1 / 4)$ & $(1,0,1)$ \\
\hline$(5 / 8,1 / 4,0)$ & $(0,1,1)$ & $(0,7 / 8,3 / 4)$ & $(1,0,-1)$ & $(0,5 / 8,1 / 4)$ & $(1,0,1)$ \\
\hline$(3 / 8,3 / 4,0)$ & $(0,1,1)$ & $(0,1 / 8,5 / 4)$ & $, 0,-1)$ & $(0,3 / 8,3 / 4)$ & $(1,0,1)$ \\
\hline$(7 / 8,3 / 4,0)$ & $(0,1,1)$ & $(0,5 / 8,5 / 4)$ & $, 0,-1)$ & $(0,7 / 8,3 / 4)$ & $(1,0,1)$ \\
\hline$(1 / 8,5 / 4,0)$ & $(0,1,1)$ & $(0,3 / 8,7 / 4)$ & $, 0,-1)$ & $(0,1 / 8,5 / 4)$ & $(1,0,1)$ \\
\hline$(5 / 8,5 / 4,0)$ & $(0,1,1)$ & $(0,7 / 8,7 / 4)$ & $(1,0,-1)$ & $(0,5 / 8,5 / 4)$ & $(1,0,1)$ \\
\hline$(3 / 8,7 / 4,0)$ & $(0,1,1)$ & $(0,-3 / 8,-7 / 4)$ & $(1,0,-1)$ & $(0,3 / 8,7 / 4)$ & $(1,0,1)$ \\
\hline$(7 / 8,7 / 4,0)$ & $(0,1,1)$ & $(0,-7 / 8,-7 / 4)$ & $(1,0,-1)$ & $(0,7 / 8,7 / 4)$ & $(1,0,1)$ \\
\hline$(-1 / 8,-5 / 4,0)$ & $(0,1,1)$ & $(0,-1 / 8,-5 / 4,0)$ & $(1,0,-1)$ & $(0,-1 / 8,-5 / 4)$ & $(1,0,1)$ \\
\hline$(-5 / 8,-5 / 4,0)$ & $(0,1,1)$ & $(0,-5 / 8,-5 / 4)$ & $(1,0,-1)$ & $(0,-5 / 8,-5 / 4)$ & $(1,0,1)$ \\
\hline$(-3 / 8,-3 / 4,0)$ & $(0,1,1)$ & $(0,-3 / 8,-3 / 4)$ & $(1,0,-1)$ & $(0,-3 / 8,-3 / 4)$ & $(1,0,1)$ \\
\hline$(-7 / 8,-3 / 4,0)$ & $(0,1,1)$ & $(0,-7 / 8,-3 / 4)$ & $(1,0,-1)$ & $(0,-7 / 8,-3 / 4)$ & $(1,0,1)$ \\
\hline$(-1 / 8,-1 / 4,0)$ & $(0,1,1)$ & $(0,-1 / 8,-1 / 4)$ & $(1,0,-1)$ & $(0,-1 / 8,-1 / 4)$ & $(1,0,1)$ \\
\hline$(-5 / 8,-1 / 4,0)$ & $(0,1,1)$ & $(0,-5 / 8,-1 / 4)$ & $(1,0,-1)$ & $(0,-5 / 8,-1 / 4)$ & $(1,0,1)$ \\
\hline$(-3 / 8,1 / 4,0)$ & $(0,1,1)$ & $(0,-3 / 8,1 / 4)$ & $(1,0,-1)$ & $(0,-3 / 8,1 / 4)$ & $(1,0,1)$ \\
\hline$(-7 / 8,1 / 4,0)$ & $(0,1,1)$ & $(0,-7 / 8,1 / 4)$ & $(1,0,-1)$ & $(0,-7 / 8,1 / 4)$ & $(1,0,1)$ \\
\hline$(-1 / 8,3 / 4,0)$ & $(0,1,1)$ & $(0,-1 / 8,3 / 4)$ & $(1,0,-1)$ & $(0,-1 / 8,3 / 4)$ & $(1,0,1)$ \\
\hline$(-5 / 8,3 / 4,0)$ & $(0,1,1)$ & $(0,-5 / 8,3 / 4)$ & $(1,0,-1)$ & $(0,-5 / 8,3 / 4)$ & $(1,0,1)$ \\
\hline$(-3 / 8,5 / 4,0)$ & $(0,1,1)$ & $(0,-3 / 8,5 / 4)$ & $(1,0,-1)$ & $(0,-3 / 8,5 / 4)$ & $(1,0,1)$ \\
\hline$(-7 / 8,5 / 4,0)$ & $(0,1,1)$ & $(0,-7 / 8,5 / 4)$ & $(1,0,-1)$ & $(0,-7 / 8,5 / 4)$ & $(1,0,1)$ \\
\hline$(-1 / 8,7 / 4,0)$ & $(0,1,1)$ & $(0,-1 / 8,7 / 4)$ & $(1,0,-1)$ & $(0,-1 / 8,7 / 4)$ & $(1,0,1)$ \\
\hline$(-5)$ & $(0,1,1)$ & $(0,-5 / 8,7 / 4)$ & $(1,0,-1)$ & $5 / 8,7 / 4)$ & $0,1)$ \\
\hline
\end{tabular}

TABLE 11. A set $S_{5}$ of diad rotations parallel to the diagonal of the faces of the cube, in the groups $I \frac{4_{1}}{g} \overline{3} \frac{2}{d}$ and $I 4_{1} 32$ 
Diad rotations parallel to the diagonals of the faces of the unit cube

\begin{tabular}{|c|c|c|c|c|c|}
\hline Point & Vector & Point & Vector & Point & Vector \\
\hline$(-5 / 4,0,3 / 8)$ & $(-1,1,0)$ & $(-3 / 4,0,1 / 8)$ & $(1,1,0)$ & $(3 / 8,-5 / 4,0)$ & $(0,-1,1)$ \\
\hline$(-5 / 4,0,7 / 8)$ & $(-1,1,0)$ & $(-3 / 4,0,5 / 8)$ & $(1,1,0)$ & $(7 / 8,-5 / 4,0)$ & $(0,-1,1)$ \\
\hline$(-1 / 4,0,3 / 8)$ & $(-1,1,0)$ & $(1 / 4,0,1 / 8)$ & $(1,1,0)$ & $(3 / 8,-1 / 4,0)$ & $(0,-1,1)$ \\
\hline$(-1 / 4,0,7 / 8)$ & $(-1,1,0)$ & $(1 / 4,0,5 / 8)$ & $(1,1,0)$ & $(7 / 8,-1 / 4,0)$ & $(0,-1,1)$ \\
\hline$(3 / 4,0,3 / 8)$ & $(-1,1,0)$ & $(5 / 4,0,1 / 8)$ & $(1,1,0)$ & $(3 / 8,3 / 4,0)$ & $(0,-1,1)$ \\
\hline$(3 / 4,0,7 / 8)$ & $(-1,1,0)$ & $(5 / 4,0,5 / 8)$ & $(1,1,0)$ & $(7 / 8,3 / 4,0)$ & $(0,-1,1)$ \\
\hline$(7 / 4,0,3 / 8)$ & $(-1,1,0)$ & $(-3 / 4,0,-3 / 8)$ & $(1,1,0)$ & $(3 / 8,7 / 4,0)$ & $(0,-1,1)$ \\
\hline$(7 / 4,0,7 / 8)$ & $(-1,1,0)$ & $(-3 / 4,0,-7 / 8)$ & $(1,1,0)$ & $(7 / 8,7 / 4,0)$ & $(0,-1,1)$ \\
\hline$(-5 / 4,0,-1 / 8)$ & $(-1,1,0)$ & $(1 / 4,0,-3 / 8)$ & $(1,1,0)$ & $(-1 / 8,-5 / 4,0)$ & $(0,-1,1)$ \\
\hline$(-5 / 4,0,-5 / 8)$ & $(-1,1,0)$ & $(1 / 4,0,-7 / 8)$ & $(1,1,0)$ & $(-5 / 8,-5 / 4,0)$ & $(0,-1,1)$ \\
\hline$(-1 / 4,0,-1 / 8)$ & $(-1,1,0)$ & $(5 / 4,0,-3 / 8)$ & $(1,1,0)$ & $(-1 / 8,-1 / 4,0)$ & $(0,-1,1)$ \\
\hline$(-1 / 4,0,-5 / 8)$ & $(-1,1,0)$ & $(5 / 4,0,-7 / 8)$ & $(1,1,0)$ & $(-5 / 8,-1 / 4,0)$ & $(0,-1,1)$ \\
\hline$(3 / 4,0,-1 / 8)$ & $(-1,1,0)$ & $(0,3 / 8,-5 / 4)$ & $(1,0,-1)$ & $(-1 / 8,3 / 4,0)$ & $(0,-1,1)$ \\
\hline$(3 / 4,0,-5 / 8)$ & $(-1,1,0)$ & $(0,7 / 8,-5 / 4)$ & $(1,0,-1)$ & $(-5 / 8,3 / 4,0)$ & $(0,-1,1)$ \\
\hline$(7 / 4,0,-1 / 8)$ & $(-1,1,0)$ & $(0,3 / 8,-1 / 4)$ & $(1,0,-1)$ & $(-1 / 8,7 / 4,0)$ & $(0,-1,1)$ \\
\hline$(7 / 4,0,-5 / 8)$ & $(-1,1,0)$ & $(0,7 / 8,-1 / 4)$ & $(1,0,-1)$ & $(-5 / 8,7 / 4,0)$ & $(0,-1,1)$ \\
\hline$(1 / 8,-3 / 4,0)$ & $(0,1,1)$ & $(0,3 / 8,3 / 4)$ & $(1,0,-1)$ & $(0,1 / 8,-3 / 4)$ & $(1,0,1)$ \\
\hline$(5 / 8,-3 / 4,0)$ & $(0,1,1)$ & $(0,7 / 8,3 / 4)$ & $(1,0,-1)$ & $(0,5 / 8,-3 / 4)$ & $(1,0,1)$ \\
\hline$(1 / 8,1 / 4,0)$ & $(0,1,1)$ & $(0,3 / 8,7 / 4)$ & $(1,0,-1)$ & $(0,1 / 8,1 / 4)$ & $(1,0,1)$ \\
\hline$(5 / 8,1 / 4,0)$ & $(0,1,1)$ & $(0,7 / 8,7 / 4)$ & $(1,0,-1)$ & $(0,5 / 8,1 / 4)$ & $(1,0,1)$ \\
\hline$(1 / 8,5 / 4,0)$ & $(0,1,1)$ & $(0,-1 / 8,-5 / 4,0)$ & $(1,0,-1)$ & $(0,1 / 8,5 / 4)$ & $(1,0,1)$ \\
\hline$(5 / 8,5 / 4,0)$ & $(0,1,1)$ & $(0,-5 / 8,-5 / 4)$ & $(1,0,-1)$ & $(0,5 / 8,5 / 4)$ & $(1,0,1)$ \\
\hline$(-3 / 8,-3 / 4,0)$ & $(0,1,1)$ & $(0,-1 / 8,3 / 4)$ & $(1,0,-1)$ & $(0,-3 / 8,-3 / 4)$ & $(1,0,1)$ \\
\hline$(-7 / 8,-3 / 4,0)$ & $(0,1,1)$ & $(0,-5 / 8,3 / 4)$ & $(1,0,-1)$ & $(0,-7 / 8,-3 / 4)$ & $(1,0,1)$ \\
\hline$(-3 / 8,1 / 4,0)$ & $(0,1,1)$ & $(0,-1 / 8,-1 / 4)$ & $(1,0,-1)$ & $(0,-3 / 8,1 / 4)$ & $(1,0,1)$ \\
\hline$(-7 / 8,1 / 4,0)$ & $(0,1,1)$ & $(0,-5 / 8,-1 / 4)$ & $(1,0,-1)$ & $(0,-7 / 8,1 / 4)$ & $(1,0,1)$ \\
\hline$(-3 / 8,5 / 4,0)$ & $(0,1,1)$ & $(0,-1 / 8,7 / 4)$ & $(1,0,-1)$ & $(0,-3 / 8,5 / 4)$ & $(1,0,1)$ \\
\hline$(-7 / 8,5 / 4,0)$ & $(0,1,1)$ & $(0,-5 / 8,7 / 4)$ & $(1,0,-1)$ & $(0,-7 / 8,5 / 4)$ & $(1,0,1)$ \\
\hline
\end{tabular}

TABLE 12. A set $S_{6} \subset S_{5}$ of diad rotations parallel to the diagonal of the faces of the unit cube that appear in the group $P 4_{1} 32$

The latter follows from the fact that $G_{z}(\pi(p))$ equals the projection of the part of $G p$ in the same horizontal plane as $p$ (for a sufficiently generic $p$ ).

That is to say: we can intersect our previously computed extended Voronoi regions $\operatorname{VorExt}_{G}\left(T_{0}\right)$ (for $T_{0} \in\left\{A_{0}, \ldots, D_{0}\right\}$ ) with $\pi^{-1}\left(\operatorname{VorExt}_{G_{z}}\left(\pi\left(T_{0}\right)\right)\right.$ ) and use the new, smaller region to compute influence regions. In order to do this we check, for each tile in $\operatorname{VorExt}_{G}\left(T_{0}\right)$, whether its projection intersects $\operatorname{VorExt}_{G_{z}}\left(\pi\left(T_{0}\right)\right)$. If it does not then we discard that tile. Needless to say that we apply this process three times to each prototile, for the three coordinate projections $X Y, X Z$ and $Y Z$.

For the groups with a planar $p 1$ this step does not reduce the regions at all, so we do not show the details. But for the other three types of groups, $p 2, p g$ and $p g g$, this step is significant. Roughly speaking, it allows us to use some glide reflections present in some of the quarter groups to cut the extended Voronoi region further.

As a planar prototile we have used a square of side $1 / 4$ for $p g$ and half of it (a right triangle) for $p g g$ and $p 2$. The reason for this difference is that in $p g$ the extended Voronoi region would not decrease significantly if we took a triangle. We omit the calculation of the extended Voronoi regions, shown in Figure 9. The details can be found in [3] and [1. Let us only say that the calculation is based on the planar version of Lemmas 2.4 and 2.5. plus the analogue for glide reflections. Roughly speaking, each glide reflection with translation vector, say $v$, "acts as if" the group $G_{z}$ contained a rotation of order two with center on the glide reflection 


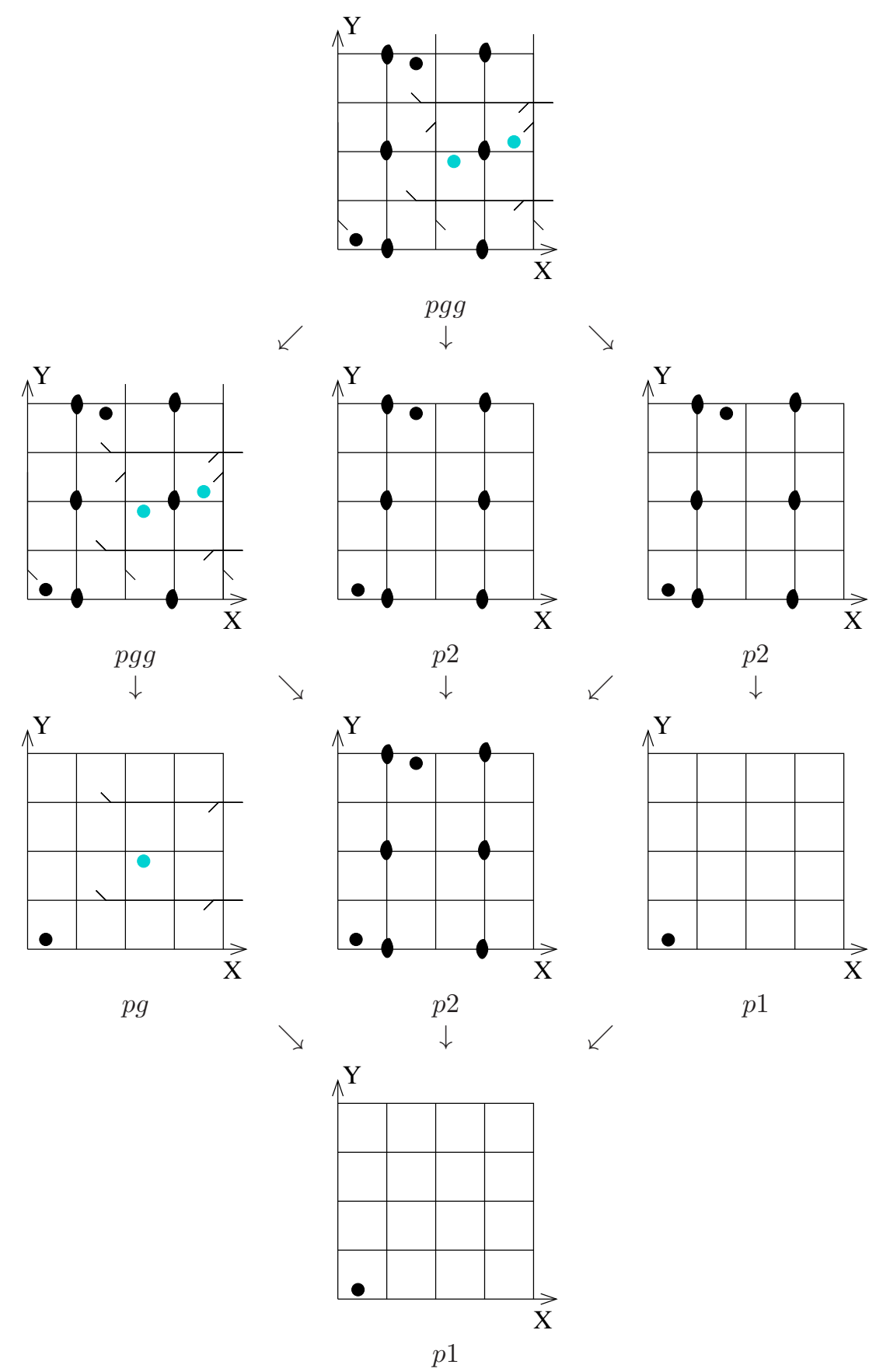

Figure 8. Plane groups of the quarter groups.

axis and at distance $|v / 2|$ from the orthogonal projection of the prototile $T_{0}$ to the axis. Glide reflections are indicated as dotted lines in the figures.

Of course, we need to check whether each prototile $T_{0}$ that we used in the $3 \mathrm{D}$ step projects to lie in one planar tile. That is, in one tile obtained from the one in Figure 9 by the action of the planar group $G_{z}$. If this is not the case then the planar extended Voronoi region we consider is going to be a union of more than 


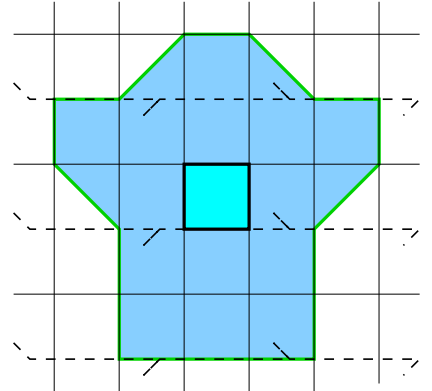

(a) plane group $p g$.

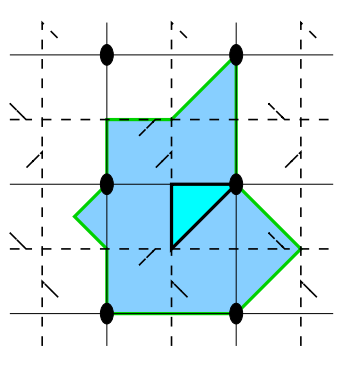

(b) plane group pgg.

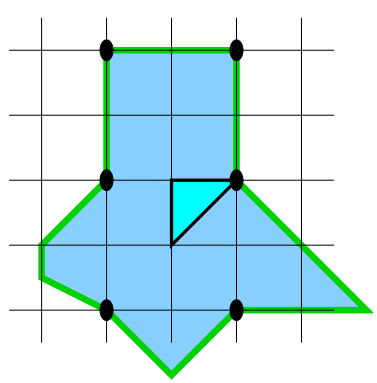

(c) plane group $p 2$.

FiguRE 9. Extended Voronoi Regions of the plane groups $p g, p g g$ and $p 2$.

one of the regions shown in the figure. The result of this check, for each of the four tiles $A_{0}, B_{0}, C_{0}$ and $D_{0}$, each of the three coordinate projections, and the three possible plane groups, is illustrated in Tables 13 and 14. As shown in the pictures, for the group $p g$ one planar tile is always enough, but for the groups $p g g$ and $p 2$ we usually need two.

3.5. Influence regions. By Theorem 2.2 once we know the influence region of, say, the prototile $A_{0}$, for a group $G$, we can obtain an upper bound for the maximum number of facets of the Dirichlet stereohedra of $G$ with base point in $A_{0}$. We only need to count how many orbit points lie in the influence region, that is, how many tiles in the $G$-orbit of $A_{0}$ are listed in our influence region. (Remember that, by construction, our influence region is a union of tiles of the auxiliary tessellation $\mathcal{T}$.) Let us see in more detail how we compute this number.

By definition, a tile $T$ is in $\operatorname{Infl}_{G}\left(A_{0}\right)$ if and only if $\operatorname{VorExt}_{G}\left(A_{0}\right) \cap \operatorname{VorExt}_{G}(T) \neq$ $\emptyset$. Note that $T$ can a priori be a tile of any of the four types, $A, B, C$ or $D$. But for $T$ produce an orbit point it is necessary (but not sufficient, see below) that $T$ be of the same type, $A$, as our prototile. Such a tile $A^{\prime}$ is in $\operatorname{Infl}_{G}\left(A_{0}\right)$ if and only if there is a tile $T^{\prime}$ (of any of the four types) in $\operatorname{VorExt}_{G}\left(A_{0}\right) \cap \operatorname{VorExt}_{G}\left(A^{\prime}\right)$.

At this point we have to clarify one feature of our encoding of tiles that is useful here. Remember that for us $\operatorname{VorExt}_{G}\left(A_{0}\right)$ is a list of tiles of $\mathcal{T}$, and that each tile $T$ of $\mathcal{T}$ has been encoded as the transformation $\rho \in \mathcal{N}(Q)$ that sends the prototile $T_{0}$ of the same type to $T$ (see Figure 10). Suppose now that $A^{\prime}$ is a tile of type $A$ in $\operatorname{Infl}_{G}\left(A_{0}\right)$. That is, $A$ is such that

$$
T^{\prime} \in \operatorname{VorExt}_{G}\left(A_{0}\right) \cap \operatorname{VorExt}_{G}\left(A^{\prime}\right)
$$

for some $T^{\prime}$. Let $\mu \in \mathcal{N}(Q)$ be the transformation that sends $A_{0}$ to $A^{\prime}$. Then, by Lemma 2.3 (and since $\mathcal{N}(Q) \subseteq \mathcal{N}(G)$ for every quarter group $G$ ), $\operatorname{VorExt}_{G}\left(A^{\prime}\right)=$ $\mu \operatorname{VorExt}_{G}(A)$. That is, there is a second tile $T_{1}$ in $\operatorname{VorExt}_{G}(A)$ such that $T^{\prime}=\mu T_{1}$. Let $\rho$ and $\rho^{\prime}$ denote the transformations that send the prototile $T_{0}$ of the appropriate type to $T^{\prime}$ and $T_{1}$, respectively. We obviously have that (see Figure 10 again):

$$
\mu=\rho^{\prime} \circ \rho^{-1} \text {. }
$$

Therefore, all the tiles $A \subset \operatorname{Infl}_{G}\left(A_{0}\right)$ that may possibly produce a facet in our Dirichlet stereohedra can be represented as the composition $\rho^{\prime} \circ \rho^{-1}$ where $\rho$ and $\rho^{\prime}$ are two isometries (that represent tiles) in $\operatorname{VorExt}_{G}\left(A_{0}\right)$.

There is a final step. Each transformation $\mu=\rho^{\prime} \circ \rho^{-1}$ obtained in this way is clearly in $\mathcal{N}(Q)$, but it may not be in our particular group $G$. Only if it is in $G$ it 


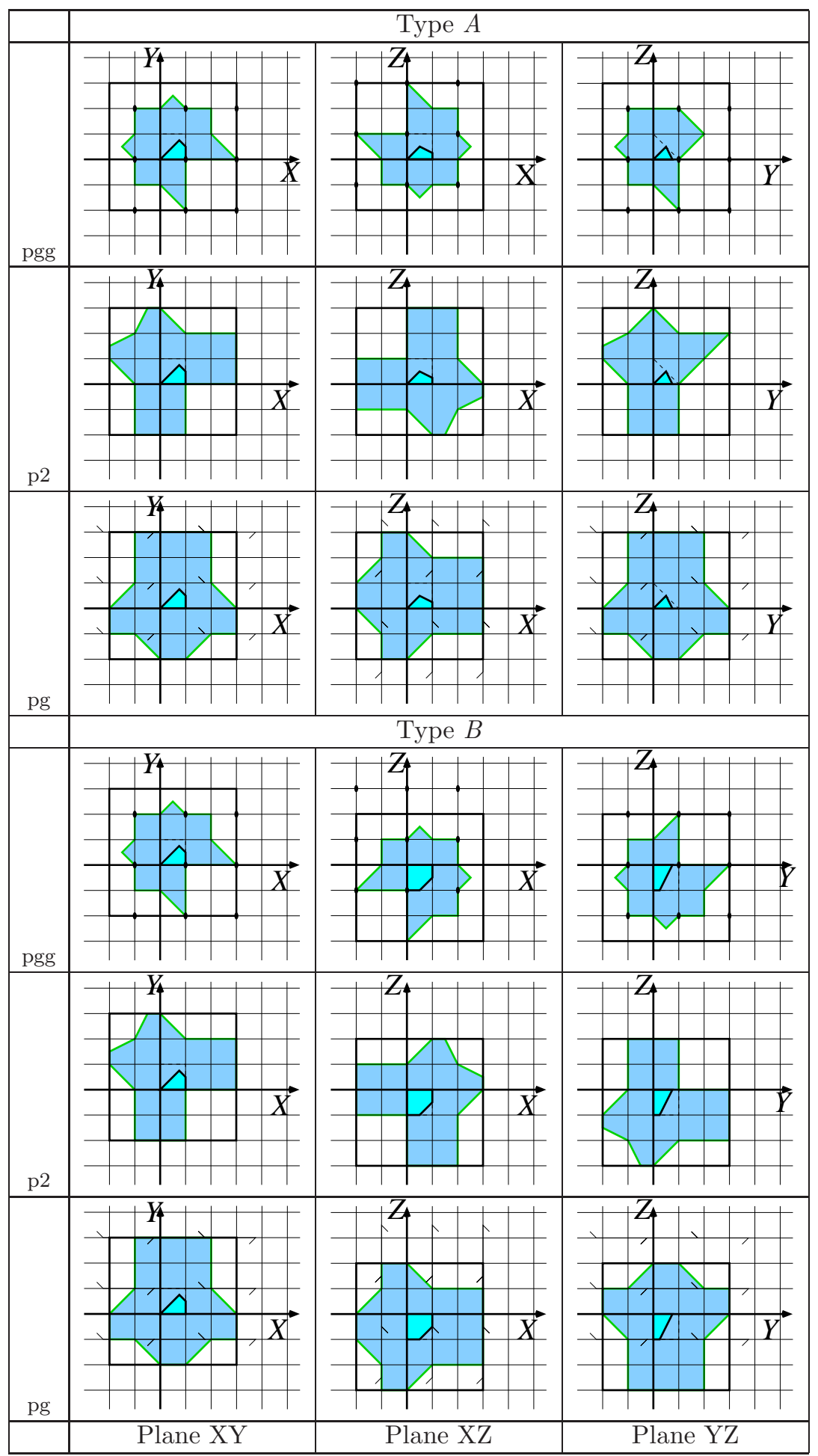

TABLE 13. Extended Voronoi Regions of the plane groups of the quarter groups. Prototiles of types $A$ and $B$

contributes one neighbor to the bound. To check this we use the coset classification of all the tiles in our initial population, shown in Table 4. 


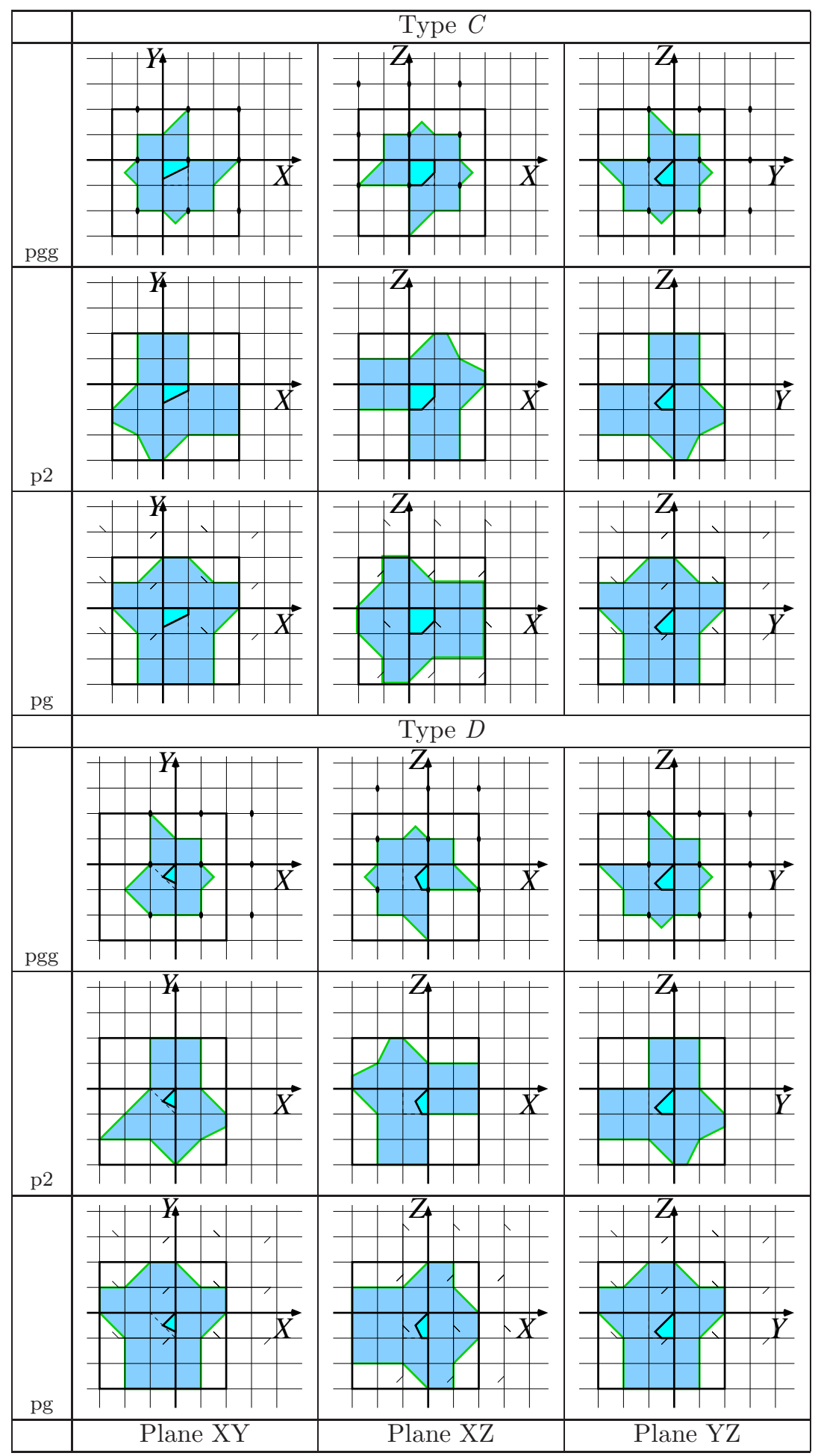

TABLE 14. Extended Voronoi Regions of the plane groups of the quarter groups. Prototiles of types $C$ and $D$ 


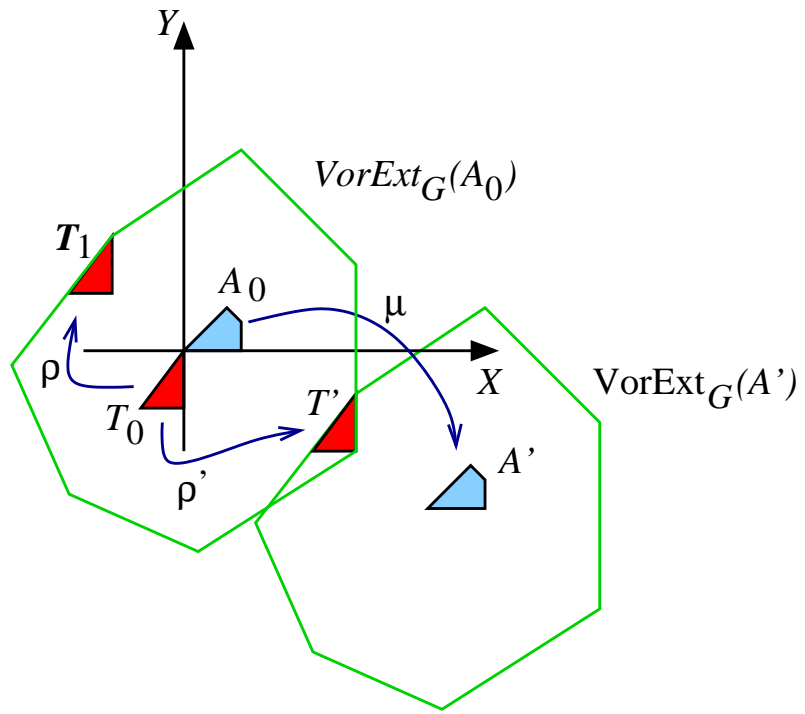

Figure 10. Calculation of the influence region

The influence regions for the three other prototiles $B_{0}, C_{0}$ and $D_{0}$ are obtained in exactly the same way. Once we have the number of elements in each influence region of $G$ for each prototile, we take the biggest of these numbers as a bound of the maximum number of facets of Dirichlet stereohedra for $G$.

The results of these calculations are shown in columns (1), (2), (3) and (4) of Table 1. For each group, the rightmost number of these three columns is the maximum size of influence regions obtained. Column (1) shows the bound obtained if we neglect the diad rotations and the planar projection step. Columns (2), (3) and (4) show how the bound decreases after considering, respectively, the coordinate diad rotations, the diagonal diad rotations, and the planar projection step.

\section{REFERENCES}

[1] D. Bochis and F. Santos. On the number of facets of 3-dimensional Dirichlet stereohedra I: groups with reflections. Discrete Comput. Geom. 25:3 (2001), 419-444.

[2] D. Bochiş and F. Santos. On the number of facets of 3-dimensional Dirichlet stereohedra II: non-cubic groups. Beitrge Algebra Geom. 47:1 (2006), 89-120.

[3] D. Bochiş. Estereoedros de Dirichlet en 2 y 3 dimensiones. Ph. D. Thesis, Universidad de Cantabria, 1999.

[4] P. Brass, W. Moser, and J. Pach. Research problems in discrete geometry, Springer-Verlag, 2005.

[5] J. H. Conway, O. Delgado Friedrichs, D.H. Huson and W.P. Thurston. On Three-Dimensional Space Groups. Contributions to Algebra and Geometry. Vol 42. No 2 (2001), 475-507.

[6] B. N. Delone (or Delaunay). A proof of the fundamental theorem of the theory of stereohedra. In Dokl. Akad. Nauk. SSSR 138 (1961), 1270-1272. (In Russian)

[7] P. Engel. Über Wirkungsbereichsteilungen von kubischer Symmetrie, Z. Krist. 154 (1981), no. 3-4, 199-215.

[8] J. Erickson. Local polyhedra and geometric graphs, Comput. Geom., 31:1-2 (2005), 101-125,

[9] J. Erickson. Nice Point Sets Can Have Nasty Delaunay Triangulations, Discrete Comput. Geom., 30:1 (2003), 109-132.

[10] J. Erickson, S. Kim. Arbitrarily large neighborly families of congruent symmetric convex 3-polytopes, in Discrete Geometry: In Honor of W. Kuperberg's 60th Birthday (András Bezdek, editor), Marcel-Dekker, 2003, pp. 267-278.

[11] B. Grünbaum and G. C. Shephard. Tilings with congruent tiles, Bull. Amer. Math. Soc. 3 (1980), 951-973. 
[12] D. Hilbert. Mathematische Probleme, in: Gesammelte Abhandlungen, Vol 3, (D. Hilbert, ed.), Springer-Verlag, Berlin, 1935, pp. 290-329.

[13] E. Koch. Wirkungsbereichspolyeder und Wirkungsbereichsteilungen zu kubischen Gitterkomplexen mit weniger als drei Freiheitsgraden. Z. Kristallogr. 138 (1973), 196-215.

[14] E. Koch. A geometrical classification of cubic point configurations. Z. Kristallogr. 166 (1984), $23-52$.

[15] E. H. Lockwood and R. H. Macmillan. Geometric Symmetry. Cambridge University Press, Cambridge, 1978.

[16] J. Milnor. Hilbert's 18 problem: on crystallographic groups, fundamental domains, and on sphere packing. In Proceedings of Symposium in Pure Mathematics of American Mathematical Society, pages 491-507. Northern Illinois University, Dekalb, Illinois, 1974.

[17] P. Sabariego and F. Santos. On the number of facets of three-dimensional Dirichlet stereohedra III: Full cubic groups. Discrete and Computational Geometry, Volume 40, Number 2, 2008, pp. 159-189.

[18] D. Schattschneider and M. Senechal. Tilings, in: Handbook of Discrete and Computational Geometry (J. E. Goodman, J. O’Rourke, eds), CRC Press, 1997, pp. 43-63.

Departamento de Matemáticas, Estadística y Computación, Universidad de CantaBRIA, 39005 SANTANDER, SPAIN

E-mail address: sabariego@gmail.com, francisco.santos@unican.es . 